\title{
Altered Memory Capacities and Response to Stress in p300/CBP-Associated Factor (PCAF) Histone Acetylase Knockout Mice
}

\author{
Tangui Maurice*,',2,3, Florian Duclot 1,2,3, Johann Meunier ${ }^{1,2,3}$, Gaëlle Naert ${ }^{1,2,3}$, Laurent Givalois ${ }^{1,2,3}$, \\ Julie Meffre $^{3,4}$, Aurélie Célérier ${ }^{1,4}$, Chantal Jacquet ${ }^{5}$, Virginie Copois ${ }^{6}$, Nadir Mechti ${ }^{6}$, Keiko Ozato $^{7}$ \\ and Céline Gongora ${ }^{6}$
}

'Université Montpellier II, Montpellier, France; ${ }^{2}$ EPHE, Paris, France; ${ }^{3}$ INSERM, U7 I 0, Montpellier, France; ${ }^{4}$ CNRS, FRE2693, Montpellier, France; ${ }^{5}$ Institut de Génétique Moléculaire de Montpellier, CNRS, UMR5535, Montpellier, France; ${ }^{6}$ CNRS, UMR5 I 60, Montpellier, France; ${ }^{7}$ Laboratory of Molecular Growth Regulation, National Institute of Child Health and Human Development, National Institutes of Health, Bethesda, MD, USA

\begin{abstract}
Chromatin remodeling by posttranslational modification of histones plays an important role in brain plasticity, including memory, response to stress and depression. The importance of H3/4 histones acetylation by CREB-binding protein (CBP) or related histone acetyltransferase, including $\mathrm{P} 300$, was specifically demonstrated using knockout (KO) mouse models. The physiological role of a related protein that also acts as a transcriptional coactivator with intrinsic histone acetylase activity, the p300/CBP-associated factor (PCAF), is poorly documented. We analyzed the behavioral phenotype of homozygous male and female PCAF KO mice and report a marked impact of PCAF deletion on memory processes and stress response. PCAF KO animals showed short-term memory deficits at 2 months of age, measured using spontaneous alternation, object recognition, or acquisition of a daily changing platform position in the water maze. Acquisition of a fixed platform location was delayed, but preserved, and no passive avoidance deficit was noted. No gender-related difference was observed. These deficits were associated with hippocampal alterations in pyramidal cell layer organization, basal levels of Fos immunoreactivity, and MAP kinase activation. PCAF KO mice also showed an exaggerated response to acute stress, forced swimming, and conditioned fear, associated with increased plasma corticosterone levels. Moreover, learning and memory impairments worsened at 6 and 12 months of age, when animals failed to acquire the fixed platform location in the water maze and showed passive avoidance deficits. These observations demonstrate that PCAF histone acetylase is involved lifelong in the chromatin remodeling necessary for memory formation and response to stress.

Neuropsychopharmacology (2008) 33, I584- 1602; doi:I0.I038/sj.npp. I 30I55I; published online 5 September 2007
\end{abstract}

Keywords: histone acetylase PCAF; short-term memory; spatial memory; stress; age

Posttranslational histone modifications exert a regulatory role during gene transcription by remodeling the structure of chromatin DNA-protein complexes (Varga-Weisz, 2005). Acetylation of specific lysine residues on $\mathrm{H} 3$ or $\mathrm{H} 4$ histones generally allows binding of the transcriptional machinery to DNA and facilitates transcription (Jenuwein and Allis, 2001). Conversely, $\mathrm{H} 3$ or $\mathrm{H} 4$ histone methylation promotes transcriptional repression (Lachner et al, 2001). Histone methylation may also facilitate DNA methylation, which in turn induces further transcriptional repression (Lachner et al, 2001). Chromatin remodeling plays an important role in several aspects of long-term cellular plasticity,

*Correspondence: Dr T Maurice, INSERM, U 7IO, EPHE, Université de Montpellier II, Place Eugène Bataillon, Montpellier, Cedex 5, 34095, France, Tel: + 330467 |4 36 23, Fax: + 330467 |4 33 86, E-mail: Tangui.Maurice@univ-montp2.fr

Received 24 April 2007; revised 5 July 2007; accepted 25 July 2007 including neuronal differentiation (Lunyak et al, 2002), seizures (Tsankova et al, 2004), learning and memory (Korzus et al, 2004; Levenson et al, 2004; Levenson and Sweatt, 2005), responses to stress and depression (Tsankova et al, 2006), drug addiction (Kumar et al, 2005), and neurodegeneration (Steffan et al, 2001; Hoshino et al, 2003). In particular, a direct effect of fear conditioning consolidation, and subsequent activation of $N$-methyl-D-aspartate receptors increased histone $\mathrm{H} 3$ acetylation in the CA1 area of the hippocampus, and this effect was blocked by inhibition of ERK signaling (Levenson et al, 2004). Moreover, chronic social defeat stress increased repressive histone methylation and subsequent treatment with the tricyclic antidepressant imipramine reversed this downregulation and increased histone acetylation (Tsankova et al, 2006). Histones posttranslational modifications, leading to chromatin remodeling, therefore appear to be a very rapid regulatory system for brain plasticity. 
Other regulators of gene expression include transcription factors, such as the cAMP-responsive element-binding protein (CREB) or its coactivator, the CREB-binding protein (CBP) (Chan and La Thangue, 2001). However, CBP, and its homolog p300, also acts as a scaffold protein by interacting with several transcription factors and components of the RNA polymerase II (Pol II) and as a histone acetyltransferase (HAT) (Ogryzko et al, 1996; Janknecht, 2002; Chan and La Thangue, 2001). Both functions are intimately linked and dynamic changes in chromatin organization directly affect gene expression. The importance of CBP and/or its HAT activity in memory consolidation was recently demonstrated using either heterozygous CBP knockout (KO) mice (Oike et al, 1999; Alarcon et al, 2004) or a mouse expressing a dominantnegative $\mathrm{CBP}\left(\mathrm{HAT}^{-}\right)$variant, in which HAT activity is abolished (Korzus et al, 2004).

A unique role, although not yet documented, could also be played by the p300/CBP-associated factor (PCAF), which also acts as a transcriptional coactivator bearing intrinsic histone acetylase activity. PCAF substrates include histones (H3 on Lys ${ }^{14}$ and $\mathrm{H} 4$ on Lys ${ }^{8}$ ), coactivators and transcription factors (Imhof et al, 1997; Munshi et al, 1998; Liu et al, 1999; Sartorelli et al, 1999; Martinez-Balbas et al, 2000). PCAF is present in a large multiprotein complex consisting of more than 20 distinct polypeptides that are able to acetylate histones in a nucleosomal context (Ogryzko et al, 1998). PCAF belongs to the Gcn5-related $\mathrm{N}$-acetyltransferase superfamily of acetyltransferase and shares close similarity with GCN5 over its entire sequence. Both proteins are present in the same complexes and GCN5 can act at the same Lys on histones $\mathrm{H} 3$ and $\mathrm{H} 4$ as PCAF, suggesting that both acetylases may have redundant biological activity.

Homozygous PCAF KO and GCN5 KO mice have been generated by replacing part of the PCAF or GCN5 gene with a neo gene cassette using homologous recombination (Yamauchi et al, 2000). Unexpectedly, these mice showed distinct phenotypes. The GCN5 homozygous KO was lethal in embryo, while PCAF KO mice displayed no obvious phenotype and no apparent developmental deficits (Yamauchi et al, 2000). GCN5 is an essential gene expressed ubiquitously and early during development, ie by day 8 post coitum (dpc). PCAF, however, is expressed later in embryonic development at $12.5 \mathrm{dpc}$, and is not essential for viability. Moreover, in early embryogenesis, PCAF and GCN5 are differentially regulated and may have distinct biological activity. Particularly, transcriptional activation by PCAF is required for nuclear receptormediated activation, the growth factor-signaled pathway, and interferon activation (Blanco et al, 1988; Xu et al, 1998; Masumi et al, 1999).

In the present study, the involvement of PCAF in learning and memory processes and stress response was examined using the PCAF KO mouse. Young 2-month-old male and female PCAF KO mice were examined through a battery of behavioral tests to assess short- and long-term, spatial, contextual, and recognition learning for memory ability and response to acute stress or hedonia for emotional response. The behavioral phenotyping was sustained by morphological and physiological examination of the hippocampal formation and stress hormone assays.

\section{MATERIALS AND METHODS}

\section{Animals}

The generation of PCAF KO mice and subsequent backcross of the CD-1 strain was previously described (Yamauchi et al, 2000). PCAF KO and wild-type (WT) littermate mice were housed in groups of 10, allowed food ad libitum, and maintained in a controlled environment $\left(22 \pm 1^{\circ} \mathrm{C}, 55 \pm 5 \%\right.$ humidity) with a $12: 12 \mathrm{~h}$ light:dark cycle. Animals were bred by heterozygous/heterozygous crossing for 5 years, representing at least 15 backcrosses. Behavior experiments for each test have been performed on WT and KO littermates. Noteworthy, parallel experiments on animals from homozygous/homozygous (WT or KO) crossing led to undistinguishable data from those obtained from heterozygous crosses. Animal's care was in accordance with the European Community Council Directive of 24 November 1986 (86609). Male mice were used at 2 and 6 months of age and female mice were used at 2 and 12 months of age, only in behavioral experiments. Mice genotypes were obtained by PCR on genomic DNA from tail tips using specific primers amplifying WT (forward 5'-TTCTAGATCTGCCGGTGTCC- $3^{\prime}$, reverse: $5^{\prime}$-CTGCCAGACCCTGTTTACAC- ${ }^{\prime}$ ), which detect the WT allele, and Neo (forward: 5'-TCGCCTTCTTG ACGAGTTCT-3', reverse: $5^{\prime}$-GGCTGCTGATCTCGTTCTT C-3') DNA sequences, which detect the neomycin cassette.

\section{Biochemical Analyses}

RNA extraction and reverse transcription. Animals were killed, the brain dissected out on ice, and the whole forebrain or the hippocampus was isolated. All tissue samples are weighed before homogenization respecting cold chain. About $60-90 \mathrm{mg}$ of hippocampal tissue or $700 \mathrm{mg}$ of forebrain tissue was lysed. The spleen was isolated and $3 \times 10^{8}$ splenocytes or $5 \times 10^{7}$ B cells were extracted and lysed. Then, tissue or cell samples were disrupted directly into a lysis buffer using Mixer Mill ${ }^{\mathbb{R}}$ MM 300 (Qiagen, Valencia, CA). Total RNA was isolated from tissue or cell lysates using RNeasy ${ }^{\circledR}$ mini Kit (Qiagen), according to the manufacturer's instructions and additional DNAse digestion was performed on all samples during the extraction process to remove genomic DNA (Rnase-Free DNase Set Protocol for DNase treatment on RNeasy ${ }^{\circledR}$ Mini spin columns, Qiagen). RNA integrity was checked by using the 2100 Bioanalyzer (Agilent Technologies, Palo Alto, CA, USA) and quantified spectrophotometrically.

Semi-quantitative real-time $R T-P C R$. Real-time PCR was performed using the LightCycler thermal cycler system (Roche Diagnostics, Basel, Switzerland) according to the manufacturer's instructions. Primer sets for PCAF were designed using Primer3 software and are available upon request. Amplification specificity was verified with melting curve analysis and loading of the PCR product on agarose gel. The quantification data were normalized with the reference gene hypoxanthine phosphorybosyl transferase (HPRT) data. Primers were designed as follows: GGCGAAG AAGCTGGAGAAAC (sense) and CCAAGTGAGAAACGTG AGCA (antisense) for PCAF; GGGAGGCCATCACATTGTG (sense) and TCCAGCAGGTCAGCAAAGAAC (antisense) for HPRT. 
Protein extractions. Mice were killed by decapitation, the brain removed, and the hippocampi dissected out on ice and immediately frozen by immersion in liquid nitrogen. For cytoplasmic proteins analysis, frozen samples were boiled during $5 \mathrm{~min}$ in $100 \mu \mathrm{l}$ of $2 \%$ sodium dodecyl sulfate (SDS, Sigma-Aldrich, France) after sonication. After centrifugation $10 \mathrm{~min}$ at $20000 \mathrm{~g}$, supernatants were recolted and protein concentration was determined with a protein assay kit, using bovine serum albumin as standard (Biorad, France). For histone extraction, all solutions and procedures were performed at $4^{\circ} \mathrm{C}$. Frozen samples were incubated for $10 \mathrm{~min}$ in $4 \mathrm{ml}$ of homogenization buffer containing $250 \mathrm{mM}$ sucrose, $25 \mathrm{mM} \mathrm{KCl}, 0.5 \mathrm{mM}$ phenylmethylsulfonylfluoride, $0.9 \mathrm{mM}$ sodium butyrate, a protease inhibitor cocktail (Roche, France), and $50 \mathrm{mM}$ Tris- $\mathrm{HCl}$ ( $\mathrm{pH}$ 7.5). Samples were homogenized with a Dounce glass homogenizer for 14 strokes. Tissue homogenates were centrifuged at $7700 \mathrm{~g}$ for $1 \mathrm{~min}$. The cytosolic fraction (supernatant) was aspired out and stored at $-80^{\circ} \mathrm{C}$. Histones were acid extracted from the nuclear fraction (pellet) in $1 \mathrm{ml}$ of $0.4 \mathrm{~N} \mathrm{H}_{2} \mathrm{SO}_{4}$ for $30 \mathrm{~min}$. Acid extracts were centrifuged at $14000 \mathrm{~g}$ for $10 \mathrm{~min}$. Histones (supernatant) were transferred to a fresh tube and precipitated with addition of trichloroacetic acid containing $4 \mathrm{mg} / \mathrm{ml}$ sodium deoxycholate (Sigma-Aldrich) for $30 \mathrm{~min}$. Precipitated proteins were collected by centrifugation at $14000 \mathrm{~g}$ for $30 \mathrm{~min}$. The supernatant was discarded and the protein pellet was washed with $1 \mathrm{ml}$ of acidified acetone $(0.1 \% \mathrm{HCl})$ for $5 \mathrm{~min}$. The proteins were collected with a centrifugation at $14000 \mathrm{~g}$ for $5 \mathrm{~min}$ and then washed a second time with $1 \mathrm{ml}$ of acetone for $5 \mathrm{~min}$. A final centrifugation at $14000 \mathrm{~g}$ for $5 \mathrm{~min}$ was performed to collect the proteins and the supernatant was discarded. The resulting purified proteins were resuspended in $10 \mathrm{mM}$ Tris $(\mathrm{pH} \mathrm{8})$ and stored at $-80^{\circ} \mathrm{C}$. Protein concentration was measured using the BCA Protein Assay Kit (Pierce, France).

Proteins analysis by western blotting. Proteins $(50 \mu \mathrm{g}$ for phospho-Erk1/2, $4 \mu \mathrm{g}$ for histone) were diluted in an equal volume of Laemmli buffer, boiled for $5 \mathrm{~min}$, and loaded on a SDS-polyacrylamide gel at $12 \%$ for phospho-Erk $1 / 2$ and $16.5 \%$ for histone. The resolved proteins were transferred to a nitrocellulose blotting membrane. The membrane was then blocked during $30 \mathrm{~min}$ at room temperature with $5 \%$ nonfat dry milk in Tris-buffered saline (TBS) $20 \mathrm{mM} \mathrm{(pH}$ 7.6) containing $0.1 \%$ Tween- 20 (TBS-T) for phospho-Erk $1 /$ 2. The membrane was incubated overnight at $4{ }^{\circ} \mathrm{C}$, rinsed for $30 \mathrm{~min}$ in TBS-T, and then incubated for $2 \mathrm{~h}$ with a horseradish peroxidase-conjugated secondary antibody for phospho-Erk1/2. For histone $\mathrm{H} 3$ acetylation analysis, all procedures were performed according to manufacturer's recommendation. Peroxidase activity was revealed by using enhanced chemiluminescence reagents. Then, intensity of peroxidase activity was semi-quantified using the Image $J^{\circledR}$ software. Results were normalized to control values (antiMAP kinase $1 / 2$ for phospho-Erk1/2, gels stained with Coomassie blue for histone).

Antibodies. The primary antibodies used, and their dilutions were as follows: anti-phospho-MAP kinase 1/2 (No. 07-467, Upstate Biotechnology, NY, USA, dilution 1:500), anti-MAP kinase 1/2 (No. 06-182, Upstate Bio- technology, dilution $1: 1000$ ), anti-acetyl histone $\mathrm{H} 3$ (Lys $^{14}$, No. 06-599, Upstate Biotechnology, dilution 1:1000), and anti-acetyl histone H4 (Lys ${ }^{5}$, Lys $^{8}$, Lys $^{12}$, Lys ${ }^{16}$, No. 06-598, Upstate Biotechnology, dilution $1: 1000$ ). The secondary antibody was horseradish peroxidase-conjugated goat anti-rabbit IGg (Sigma-Aldrich, dilution $1: 2000$ ).

\section{Morphological Analyses}

Cresyl violet staining. Each mouse was anesthetized with sodium pentobarbital ( $100 \mathrm{mg} / \mathrm{kg}$ i.p.) and quickly transcardially perfused with $50 \mathrm{ml}$ of phosphate-buffered saline (PBS) solution ( $\mathrm{pH} 7.2$ ) followed by $50 \mathrm{ml}$ of PBS solution containing $4 \%$ paraformaldehyde $(\mathrm{w} / \mathrm{v})$. Brains were removed and kept overnight in the fixative solution. They were cut in coronal sections $(50 \mu \mathrm{m}$ thickness $)$ using a vibratome (Leica VT1000 S). Serial sections were selected to include the hippocampal formation and placed in gelatincoated slides. Sections were stained with $0.2 \%$ cresyl violet reagent (Sigma-Aldrich, France), then dehydrated with graded ethanol, treated with xylene, and mounted with DePeX medium (BDH Laboratory Supplies, UK). Examination of the CA1 area was performed using a light microscope (Dialux 22, Leica, Germany), and the slices were digitalized through a CCD camera (Sony XC-77CE, Japan) with the NIH Image v1.63 software, to facilitate processing of cell layer thickness measurements and pyramidal cells counts. Three subregions were examined, ie CA1, CA3, and the dentate gyrus. The median width was calculated from three measures on one slice and three to eight slices were counted per region per mouse. The results are presented as mean \pm SEM and analyzed using the Student's $t$-test.

Fos protein immunohistochemistry. After transcardiac perfusion with paraformaldehyde $4 \%$ and $24 \mathrm{~h}$ postfixation, coronal sections ( $50 \mu \mathrm{m}$ thickness) were cut to include the rostral part of the hippocampus and incubated for $10 \mathrm{~min}$ with $1 \% \mathrm{H}_{2} \mathrm{O}_{2}$ to inhibit endogenous peroxidase activity, and then with a Fos antibody (SC-52, Santa Cruz, CA, USA; dilution 1:5000) for $48 \mathrm{~h}$ in a PBS containing $2 \%$ bovine serum albumin (Sigma-Aldrich) and $0.1 \%$ Triton X-100 (Sigma-Aldrich), and then with a peroxidase-conjugated goat anti-rabbit IgG for $2 \mathrm{~h}$ (Jackson ImmunoResearch Europe, UK; dilution 1:500). Slices were rinsed at each step with PBS, mounted after dehydration and observed under a light microscope. Fos immunostaining was measured with a microcomputer imaging device (SAMBA ${ }^{\circledR}$ analysis Software, Alcatel, France) capturing the image through a CCD camera connected to a light microscope (Axioskop, Zeiss, Germany). Immunoreactive cells were detected within the granular cell layer, counted as number of stained cells expressing Fos, and standardized via NIH image analysis. The results, mean $\pm S E M$, were analyzed using Newman-Keuls test.

Plasma corticosterone assay. Plasma corticosterone was assayed, as previously reported (Givalois et al, 2004), with a radioimmunoassay kit (Biotrak, Amersham Biosciences, Saclay, France) in a $50 \mu \mathrm{l}$ plasma sample diluted $(1: 5)$ with the assay buffer and heated to $60^{\circ} \mathrm{C}$ for $30 \mathrm{~min}$ to displace corticosterone to the cortisol-binding protein according to 
the manufacturer's instructions. The intra- and interassay coefficients of variation were 5 and $7 \%$, respectively. The assay sensitivity was $0.6 \mathrm{ng} / \mathrm{ml}$. The results, mean \pm SEM, were analyzed using Newman-Keuls test.

\section{Behavioral Testing}

Open-field behavior. The general motility of mice was examined in a circular wooden arena (diameter $75 \mathrm{~cm}$ ). Two concentric circles were drawn on the floor (diameter 15 and $45 \mathrm{~cm}$, respectively), with the outer ring being divided into eight partitions and the middle ring into four partitions. The open-field session consisted of placing the mouse in the center circle and monitoring its movements for $10 \mathrm{~min}$ using a video camera. The following parameters were evaluated: (1) the time taken to move out of the center circle; (2) locomotion activity, in terms of number of partitions crossed, then calculated as the distance traveled (m); (3) immobility duration; (4) locomotor speed; (5) locomotion activity in the five central partitions; (6) rearing frequency; (7) grooming frequency; and (8) number of defecations. The results were analyzed at 2 months of age using two-way ANOVA (F value), with substrain and gender as independent factors, group comparisons using the Newman-Keuls test and the Student's $t$-test for the groups at 6 or 12 months of age.

Spatial learning in the water maze. The water maze was a circular pool (diameter $170 \mathrm{~cm}$, height $40 \mathrm{~cm}$ ). The water temperature, $23 \pm 1^{\circ} \mathrm{C}$, light intensity, external cues in the room, and water opacity were rigorously reproduced. A transparent plexiglas nonslippery platform (diameter $10 \mathrm{~cm}$ ) was immersed under the water surface during acquisition. Swimming was recorded using Videotrack ${ }^{\mathbb{R}}$ software (Viewpoint, Champagne-au-Mont-d'Or, France), with trajectories being analyzed as latencies and distances. The software divided the pool into four quadrants. Training consisted of three swims per day for 7 days (9 for 12-monthold mice), with $15 \mathrm{~min}$ intertrial time. Start positions, set at each limit between quadrants, were randomly selected and each animal was allowed a $90 \mathrm{~s}$ swim to find the platform. It was left $20 \mathrm{~s}$ on the platform. The median latency, expressed as mean \pm SEM, was calculated for each training day and analyzed over trials using a repeatedmeasures ANOVA (F values).

A probe test was performed $1 \mathrm{~h}$ after the last swim on day 7 or 9. The platform was removed and each animal was allowed a free $60 \mathrm{~s}$ swim. The start position for each mouse corresponded to one of two positions remote from the platform location in counterbalanced order. The platform quadrant was termed the training $(\mathrm{T})$ quadrant and others opposite $(\mathrm{O})$, adjacent right (AR), and adjacent left (AL) during retention. The percentage of time spent in each quadrant was determined. Data were analyzed using a Dunnett's test after a one-way ANOVA (F values).

A procedure for specifically assessing the short-term memory component was then performed for 3 days. The platform location changed every day but not among trials. Each animal was allowed five trials per day, with a $2 \mathrm{~min}$ intertrial time interval. Data represent the mean performance over days for each trial. Escape latencies were analyzed using a repeated-measures ANOVA (F values), followed by a Dunnett's post hoc test.

Spontaneous alternation in the $Y$ maze. The maze was made of black painted wood. Each arm was $40 \mathrm{~cm}$ long, $13 \mathrm{~cm}$ high, $3 \mathrm{~cm}$ wide at the bottom, $10 \mathrm{~cm}$ wide at the top, and converging at an equal angle. Each mouse was placed at the end of one arm and allowed to move freely through the maze during an $8 \mathrm{~min}$ session. The series of arm entries, including possible returns into the same arm, was recorded using an Apple IIe computer. An alternation was defined as entries into all three arms on consecutive occasions. The number of maximum alternations was therefore the total number of arm entries minus two and the percentage of alternation was calculated as (actual alternations/maximum alternations) $\times 100$. The data were analyzed using twoway ANOVA ( $F$ value), with substrain and gender as independent factors, and group comparisons made using Newman-Keuls test.

Object recognition procedure. The apparatus consisted of a squared open field $(1 \times 1 \times 0.4 \mathrm{~m}$ high $)$, made of dark gray plastic. Lighting was provided indirectly by a $60 \mathrm{~W}$ bulb positioned $2 \mathrm{~m}$ above the floor. The open field was surrounded by white curtains $(2 \mathrm{~m}$ high) to provide a visually uniform experimental environment, except for a striped pattern attached on the wall opposite to the position where the animals were initially placed. Objects could be placed at four defined positions, which differed by their shape and texture: (A) a black plastic chair foot cover; (B) a circular plastic box; (C) a transparent glass $25-\mathrm{ml}$ erlen vial; (D) a metallic toy battery; and (E) a plastic $50 \mathrm{ml}$ vial (Falcon). Animals' movements were quantified by videotracking, in terms of distance and duration of exploration (Videotrack, ViewPoint). An experienced experimenter quantified the number of contacts with objects, defined as the mouse's snout actually touching the object. Mice were submitted to seven 6-min sessions, with an intertrial time interval of $3 \mathrm{~min}$. For session 1, the open field was empty and animals were allowed a free exploration session to evaluate their locomotor activity, reaction to novelty, and anxiety. For sessions 2-4, the four objects A-D were placed in the open field and exploration was assessed through quantification of contacts with objects and locomotor activity. For sessions 5-6, objects $\mathrm{C}$ and $\mathrm{D}$ were inverted, to evaluate the reaction to spatial change. For session 7 , a novel object, $\mathrm{E}$, replaced the familiar object $\mathrm{A}$, to measure the reaction to novelty. The starting position was unchanged throughout the sessions, and animals were tested in a semi-randomized order. Data were analyzed using multiple comparison one-way or two-way ANOVA, using session, object, gender, or substrain as independent factors, as specified. ANOVA was followed by the Newman-Keuls post hoc comparison test.

Step-down type passive avoidance test. The apparatus consisted of a transparent acrylic cage $(30 \times 30 \times 40 \mathrm{~cm}$ high) with a grid floor, inserted in a soundproof outer box $(35 \times 35 \times 90 \mathrm{~cm}$ high $)$. The cage was illuminated with a $15 \mathrm{~W}$ lamp. A wooden platform $(4 \times 4 \times 4 \mathrm{~cm})$ was fixed at the center of the grid floor. Electric shocks $(1 \mathrm{~Hz}, 500 \mathrm{~ms}$, 
$43 \mathrm{~V} \mathrm{DC})$ were delivered to the grid floor using an isolated pulse stimulator (Model 2100, AM Systems, Everett, WA, USA). The test involved two training sessions, at 90-min time interval, and in a retention session carried out $24 \mathrm{~h}$ after the first training. Each mouse was placed on the platform during the training sessions. When it stepped down and placed its four paws on the grid floor, shocks were delivered for $15 \mathrm{~s}$. Step-down latency and the numbers of vocalizations and flinching reactions were measured. Shock sensitivity was evaluated by summing these last two numbers. Animals that did not step down within $60 \mathrm{~s}$ during the second session were considered as remembering the task and taken off without receiving more shocks. The retention test was performed similarly as training, but with no shock. Each mouse was placed again on the platform and the step-down latency was recorded with a cutoff of $300 \mathrm{~s}$. Two parametric measures of retention were analyzed: the latency and the number of animals reaching an avoidance criterion, defined as correct if the retention latency was higher than threefold the second training latency and at least greater than $60 \mathrm{~s}$. Latencies did not show a normal distribution, since cutoff times were set, and were thus expressed as median and interquartile range. They were analyzed using the Kruskal-Wallis nonparametric ANOVA (KW values), followed by a Dunn's test.

Forced swimming test. Each mouse was placed in a glass cylinder (diameter $12 \mathrm{~cm}$, height $24 \mathrm{~cm}$ ) filled with water at a height of $12 \mathrm{~cm}$. Water temperature was maintained at 22$23^{\circ} \mathrm{C}$. The animal was forced to swim for $15 \mathrm{~min}$ on the first day. On the second day, each mouse was placed again in the water and forced to swim for $6 \mathrm{~min}$. The sessions were videotaped and duration of immobility analyzed minute per minute. The mouse was considered as immobile when it stopped struggling and moved only to remain floating in the water. Results were analyzed using multiple comparison two-way ANOVA, using time (minute or day) and substrain as independent factors, followed by a Newman-Keuls post hoc comparison test.

Conditioned fear stress. The apparatus was a transparent acrylic rectangular cage $(30 \times 30 \times 40 \mathrm{~cm}$ high $)$ equipped with a metal wire floor. On day 1 , each mouse was placed in the test cage and received intermittent electric shocks $(0.1 \mathrm{~Hz}, 200 \mathrm{~ms}, 60 \mathrm{~V} \mathrm{DC})$ for $10 \mathrm{~min}$ through an isolated pulse stimulator (Model 2100, AM Systems). The test session was performed after $24 \mathrm{~h}$. Animals were placed again in the cage, but no foot shock was delivered. The ambulatory activity of mice during each session was measured for 6 min using an infrared beam activity device (Opto-varimex, Columbus Instruments, Columbus, $\mathrm{OH}$, USA), in which the cage was inserted. The nonshocked control group was operated similarly, but received no shock. Results were analyzed using multiple comparison two-way ANOVA, with time (minute or day) and substrain as independent factors, followed by a Newman-Keuls post hoc comparison test.

Measure of sucrose preference (anhedonia). Animals were placed in cages of four individuals 1 week before the test and were water deprived $16 \mathrm{~h}$ before each test. They were presented two weighted bottles of fluid, one with tap water, the other one with a sucrose $4 \%$ solution, for $2 \mathrm{~h}$. The bottles were weighed after $1 \mathrm{~h}$ and at the end of the test to evaluate the mean fluid intake per hour per mouse. They were then allowed free access to tap water for $6 \mathrm{~h}$. The results showed that the contribution of the second hour could be neglected and therefore only the first hour consumption is presented. The test was performed daily for 9 days. Data were analyzed using multiple comparison two-way ANOVA, using day and substrain as independent factors.

\section{RESULTS}

\section{Brain PCAF Expression and Histone Acetylation in PCAF KO Mice}

The absence of expression of PCAF was initially checked by genotyping. This was carried out on DNA extracted from mouse tail (Figure 1a). The absence of PCAF expression was then investigated using RNA extracts from WT and KO animals. Figure $1 \mathrm{~b}$ indicates that PCAF is expressed in all tissues tested in WT animals (B cells, splenocytes, hippocampus, and brain) and that no PCAF expression was observed in $\mathrm{KO}$ animals, as expected. Moreover, semiquantitative real-time RT-PCR analyses were unable to detect PCAF expression in KO mice in hippocampus, as shown in Figure 1c. We also examined the level of acetylation of histone $\mathrm{H} 3$ on $\mathrm{Lys}^{14}$ and $\mathrm{H} 4$ on Lys ${ }^{8}$, which are known targets of PCAF, in the hippocampus. The levels of histone $\mathrm{H} 3$ and $\mathrm{H} 4$ acetylation were not diminished in PCAF KO mice compared to WT mice (Figure 1d and e).

\section{Short-Term Memory Impairment in PCAF KO Mice at 2 Months of Age}

Different batches of animals were used for behavioral phenotyping and morphological analyses, with animals undergoing behavioral tests in series or not, as detailed in the Supplementary Figure 1.

Prior to any behavioral investigation, we first examined the general motility of female and male WT and PCAF KO mice in an open-field paradigm. As summarized in Table 1, two-way ANOVA analyses, with gender and substrain as independent factors, revealed no significant variations between WT and PCAF KO animals for any of the parameters examined. These observations concerning locomotion, exploration, or anxiety behaviors indicated that the animals did not show any general behavioral deficits that would have impeded the validity of the memory tests.

We then analyzed the behavioral consequences of inactivation of PCAF expression in a series of memory tasks involving spatial and associative learning. Female and male animals were examined in parallel.

Hippocampus-dependent learning abilities were examined using the Morris water-maze task. Animals were first submitted to repeated acquisition of a fixed platform location, ie a procedure involving short-term and reference memory components. For both PCAF KO and WT female mice, latencies in finding the platform decreased over the acquisition-training period. The statistical analysis showed 


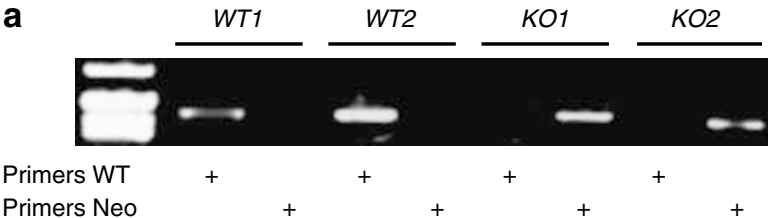

b

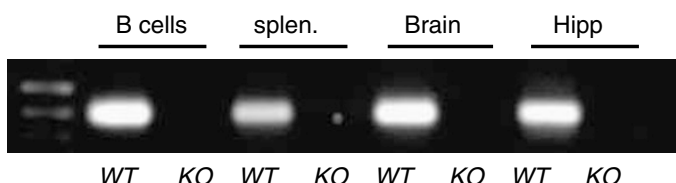

$\begin{array}{llllllll}W T & K O & W T & K O & W T & K O & W T & K O\end{array}$

d

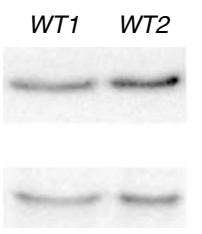

KO1 KO2
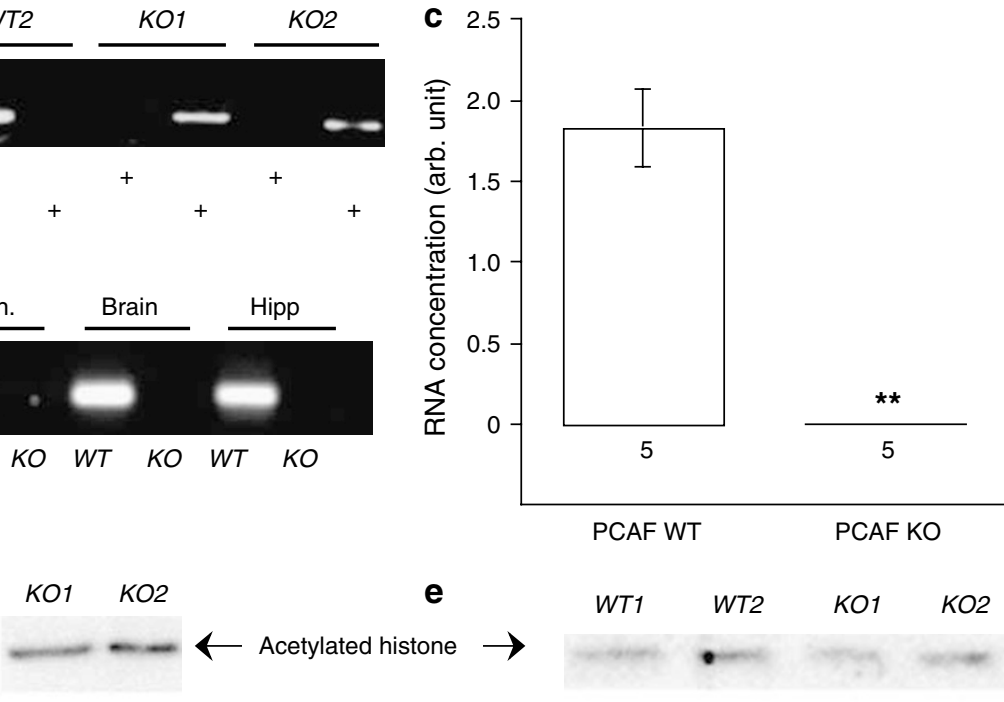

PCAF WT

PCAF KO
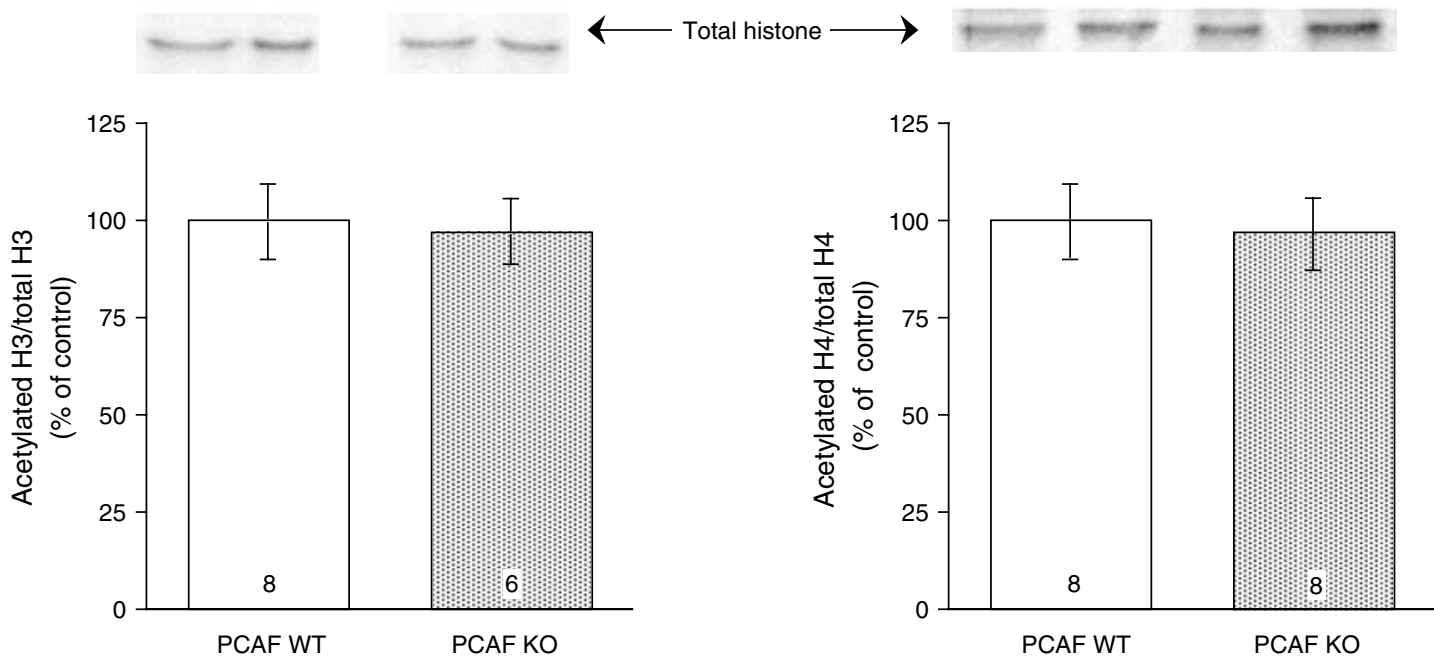

Figure I Two-month-old p300/CBP-associated factor (PCAF) knockout (KO) mice showed absence of PCAF but preserved histone acetylation. Mice genotypes were performed by PCR on genomic DNA from tail for wild-type (WT) and KO animals (a). Representative PCR analyses for PCAF expression in

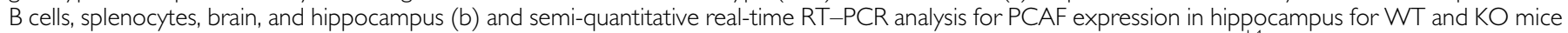

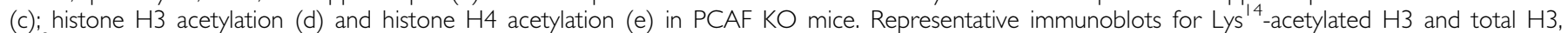

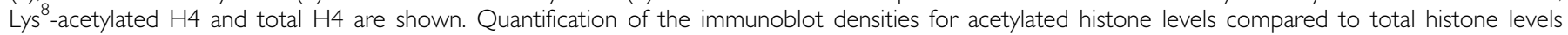
was performed on the number of animals indicated within the columns. $* * * 0.01$ vs WT group, Student's t-test.

significant effects in WT and PCAF KO mice (Figure 2a). Latencies shown by PCAF KO animals were augmented as compared with WT animals and significant differences were measured on days 2 and 4 (Figure 2a). During the probe test, performed $1 \mathrm{~h}$ after the last trial, both WT and PCAF $\mathrm{KO}$ animals swam preferentially in the $\mathrm{T}$ quadrant during the 60 -s session (Figure 2b). The time spent in this quadrant was similar between both groups, representing about $41 \%$, indicating that they both correctly learned the platform location to an equal extent.

Male animals also showed correct learning abilities, since latencies to find the platform decreased over the acquisition-training period for WT or PCAF KO mice (Figure 2c). Latencies shown by PCAF KO animals appeared to be higher than that observed for WT, with significant differences measured during days 2, 3, and 5 (Figure 2c). During the probe test, both WT and PCAF KO mice swam preferentially in the $\mathrm{T}$ quadrant during the 60 -s session (Figure 2d). The time spent in this quadrant was similar between both groups, representing about $51 \%$, indicating that they both correctly learned the location of the hidden platform to an equal extent.

Swimming speeds were calculated among groups and were $29.4 \pm 1.2 \mathrm{~cm} / \mathrm{s}$ for female WT, $26.9 \pm 0.9 \mathrm{~cm} / \mathrm{s}$ for female PCAF KO, $25.5 \pm 1.5 \mathrm{~cm} / \mathrm{s}$ for male $\mathrm{WT}$, and $23.3 \pm 1.2 \mathrm{~cm} / \mathrm{s}$ for male PCAF KO. The two-way ANOVA indicated a significant effect for gender $\left(\mathrm{F}_{(1,31)}=9.18\right.$, $p<0.01)$ but not for substrain $\left(F_{(1,31)}=3.62, p>0.05\right)$ or gender $\times$ substrain $(\mathrm{F}<1)$.

The spatial short-term memory was then estimated by changing the platform location daily for 3 training days. The first trial of each training day was an informative sample trial wherein the animal was allowed to swim to the platform at its new location. As shown in Figure 2e and f, 
Table I Behavioral Parameters of 2-Month-Old Female and Male PCAF KO and WT Mice in the Open-Field Test

\begin{tabular}{|c|c|c|c|c|}
\hline \multirow[b]{2}{*}{ Parameter } & \multicolumn{2}{|c|}{ Female } & \multicolumn{2}{|c|}{ Male } \\
\hline & WT & PCAF KO & WT & PCAF KO \\
\hline Weight (g) & $22.7 \pm 0.9$ & $22.0 \pm 0.6$ & $29.1 \pm 0.9$ & $27.9 \pm 1.1$ \\
\hline Latency to start (s) & $5 \pm 2$ & $10 \pm 3$ & $15 \pm 5$ & $16 \pm 4$ \\
\hline Locomotion (m) & $17.2 \pm 1.6$ & $13.8 \pm 2.1$ & $19.6 \pm 2.9$ & $18.3 \pm 3.2$ \\
\hline $\begin{array}{l}\text { Locomotion in } \\
\text { center }(\%)\end{array}$ & $15.5 \pm 3.9$ & $9.1 \pm 2.6$ & $15.3 \pm 2.7$ & $18.0 \pm 4.8$ \\
\hline Speed (m/min) & $2.56 \pm 0.13$ & $2.10 \pm 0.17$ & $2.66 \pm 0.31$ & $2.54 \pm 0.25$ \\
\hline Immobility (s) & $196 \pm 20$ & $210 \pm 34$ & $139 \pm 25$ & $146 \pm 38$ \\
\hline Rearing & $10 \pm 3$ & $8 \pm 3$ & $15 \pm 6$ & $15 \pm 5$ \\
\hline Grooming & $6 \pm 1$ & $5 \pm 1$ & $6 \pm 1$ & $5 \pm 1$ \\
\hline Defecations & $4 \pm 1$ & $5 \pm 1$ & $5 \pm 1$ & $5 \pm 1$ \\
\hline$n$ & 7 & 8 & 9 & 8 \\
\hline
\end{tabular}

Abbreviations: $\mathrm{KO}$, knockout; PCAF, p300/CBP-associated factor; WT, wild type.

Results were analyzed using a two-way ANOVA, with substrain and gender as independent factors and no behavioral parameters showed significant variations.

mice exhibited a progressive decrease in escape latency over the five trials. This decrease assessed the quality of spatial short-term memory. Female WT mice showed a significant decrease in escape latencies over the swimming trials (Figure 2e). In particular, the latencies showed during trials 4 and 5 were significantly lower as compared to the first trial. Female PCAF KO mice failed to show a significant decrease in latency (Figure 2e). During trials 4 and 5, the latencies measured for PCAF KO animals were significantly higher as compared to control animals. Male WT mice showed a significant decrease in escape latencies over the swimming trials (Figure 2f). In particular, the latencies showed during trials 3-5 were significantly lower as compared to the first trial. Male PCAF KO mice failed to show a significant decrease in latency (Figure 2f). The latency measured during the last two trials for PCAF KO animals was significantly higher as compared to WT animals.

Spatial working memory was examined using the spontaneous alternation procedure in the $\mathrm{Y}$ maze, a nonaversive task allowing to differentiate between mnesic and locomotor behaviors. Both male and female PCAF KO mice showed a highly significant impairment of the alternation percentage (Figure 3a), with no difference in terms of number of arms entered during the session (Figure $3 b$ ).

Male PCAF KO mice were tested for object recognition memory. Object-oriented exploratory activity and habituation was examined between sessions 2 and 4 by measuring the contact with four objects placed within the arena (Figure 3c). The two-way ANOVA analysis revealed a significant effect for sessions and substrain, but not for the interaction. PCAF KO mice showed a significantly higher level of interaction with objects for each session as compared to WT controls, but habituation persisted throughout the sessions (Figure 3c), with a significant difference between session 2 and 4 . The reaction to spatial change was examined by inverting the place of two objects before session 5 , and measuring the contacts with objects between nondisplaced and displaced objects during sessions 5 and 6 (Figure 3d). ANOVA analyses were performed using displaced $v s$ nondisplaced objects, with sessions and group treatments as independent factors. Significant effects were measured for object, session, and substrain, but also for the object $\times$ session interaction, object $\times$ substrain, and session $\times$ substrain (Figure 3d). Post hoc comparisons showed that the control group presented preferential exploration of displaced objects as compared with nondisplaced objects during sessions 5 and 6, with significant habituation for the displaced object exploration during the last session. PCAF KO animals showed a higher level of interaction with nondisplaced objects and no difference between displaced and nondisplaced objects or between sessions (Figure 3d). Reaction to novelty was assessed by exploration of a novel object during session 7 (Figure 3e). A significant effect was observed for object and substrain, but not for the object $\times$ substrain interaction. Control animals showed a highly significant preferential exploration of the novel object, and this trend also seemed to apply to PCAF KO animals, even though a higher level of contact with familiar objects was still measured (Figure 3e).

Associative long-term memory was examined using a step-down type passive avoidance task. Two memory parameters were quantified, ie step-down latency and the percentage of animals reaching the avoidance criterion. The statistical analyses failed to show any significant effect for latency (Figure 3f) or the percentages of animals to criterion (Figure 3g).

\section{Hippocampal Morphology in PCAF KO Mice}

An examination of the brain morphology was conducted using cresyl violet staining. In particular, the pyramidal neuronal CA1/3 layers and the dendate gyrus in the hippocampal formation were analyzed, as detailed in Figure 3. Male PCAF KO mice, at 2 months of age, showed subtle modifications in hippocampal formation as compared to WT animals (Figure $4 \mathrm{a}$ and $\mathrm{b}$ ). In particular, we observed, in the pyramidal neuronal layer in the CA1 area, a moderate but significant decrease in the cell number and an increase in the layer thickness, as shown for typical slices in Figure $4 \mathrm{c}$ and $\mathrm{d}$ and averaged in Figure 4e. This indicated a decrease in cell density. In the CA3 area, a similar trend was observed (Figure $4 \mathrm{f}$ and $\mathrm{g}$ ), but no significant difference was measured (Figure $4 \mathrm{~h}$ ). The morphological aspect of the dendate gyrus was similar between WT and PCAF KO animals (Figure 4i-k).

\section{Neuronal Activation in PCAF KO Mice}

The behavioral data suggested perturbations in normal synaptic function or neuronal activation as a basis for the short-term memory deficits in PCAF KO mice. We therefore examined two physiologic responses in PCAF KO animals subjected to different behavioral paradigms. First, c-fos induction in basal conditions and after completion of the object recognition task was examined in the hippocampal formation by Fos protein immunohistochemistry (Figure 5). Second, the induction of MAP kinases, measured 

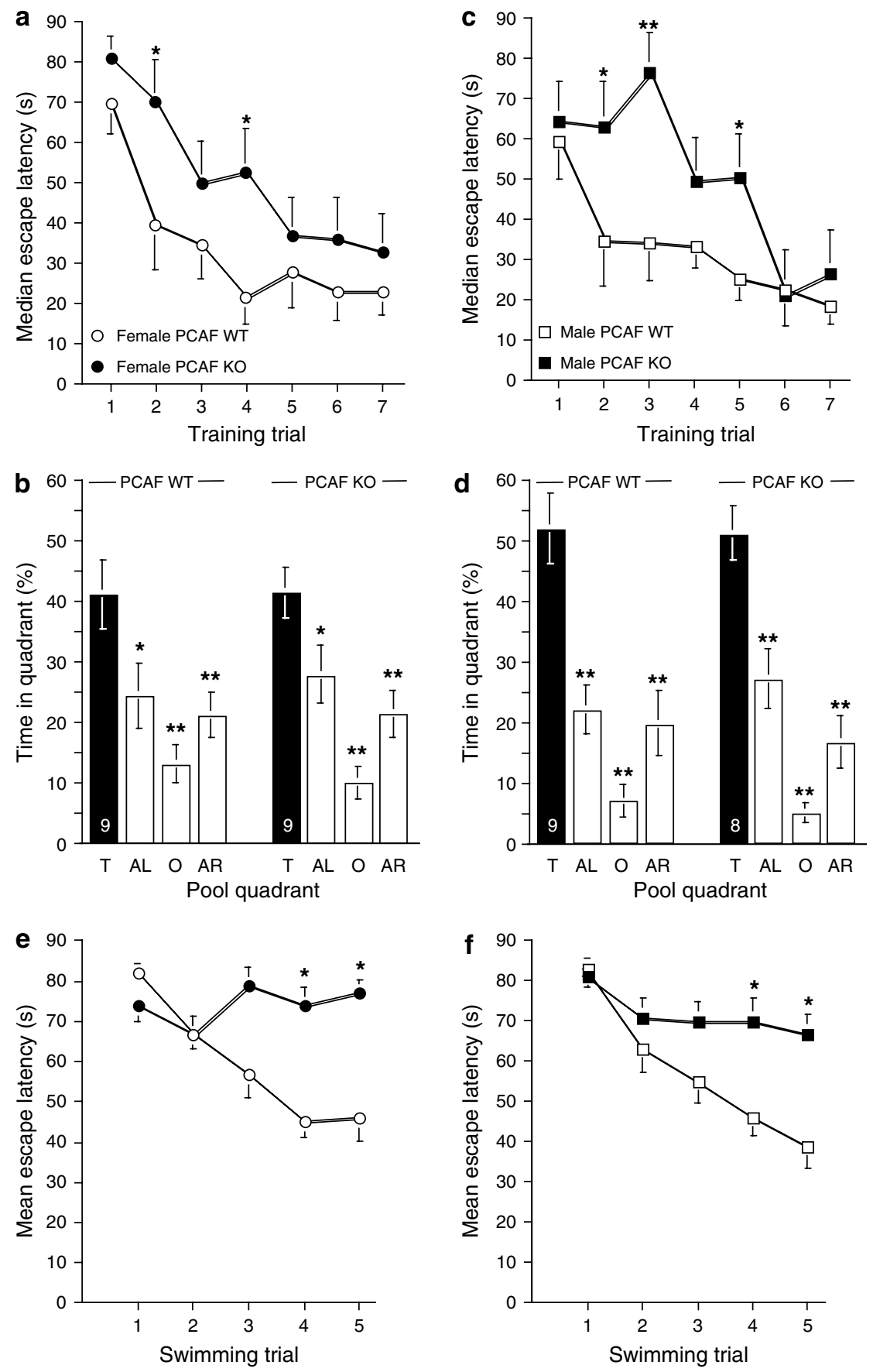

Figure 2 p300/CBP-associated factor (PCAF) knockout (KO) mice showed a preserved reference but impaired working memory for place learning in the water maze. Two-month-old female (left) and male (right) wild-type (WT) and PCAF KO mice acquired a fixed platform position in the water-maze test: acquisition profiles showing swimming duration ( $a$ and $c$ ); probe test performances (b and $d$ ). Animals were submitted for 7 days to three swims per day, with a 10 min intertrial time interval. Acquisition profiles are shown in terms of median swimming latency (a and $c)$. ANOVA: $F_{(6,62)}=7.70, p<0.000$ I for female WT; $F_{(6,62)}=5.50, p<0.00$ I for female PCAF KO mice; $F_{(6,62)}=3.60, p<0.0$ I for male $W T ; F_{(6,55)}=6.56$, $p<0.0001$ for male PCAF KO mice. ${ }^{*} p<0.05$, $* * * 0.0$ I vs the control value during the same training trial (Dunnett's test). The platform was removed and animals were submitted to a $60 \mathrm{~s}$ swim I h after the last swimming trial. The presence in each quadrant was measured. Quadrants: T, training; AL, adjacent left; $O$, opposite; $A R$, adjacent right. The number of animals per group is indicated within the T column. ANOVA: $F_{(3,35)}=7.67, p<0.00$ I for female WT; $F_{(3,35)}=13.80$, $p<0.000$ I, for female PCAF KO; $F_{(3,35)}=20.27, p<0.000$ I for male WT; $F_{(3,31)}=28.86, p<0.0001$, for male PCAF KO mice. $* p<0.05$, *** $p<0.0$ I vs the time spent in the $T$ quadrant (Dunnett's test). The short-term memory component was measured in the water maze using a daily changing platform position procedure: swimming latency profiles among the five trials, averaged over the 3 days, for female $(e)$ and male $(f) W T$ and PCAF KO mice. ANOVA: Fr $=17.35, p<0.0$ I for female WT; $\mathrm{Fr}=4.10, p>0.05$ for female PCAF KO; $\mathrm{Fr}=14.42$, $p<0.01$ for male WT; $\mathrm{Fr}=3.25, p>0.05$ for male PCAF KO. *p $<0.05 \mathrm{vs}$ the control value (Dunn's test).

through phosphorylation of ERK1/2, was examined in WT and PCAF KO mice after the passive avoidance procedure (Figure 6).
The Fos-immunopositive cells were measured in the hippocampal CA1 (Figure 5a-e), CA3 (Figure $5 \mathrm{f}-\mathrm{j}$ ), and dendate gyrus areas (Figure $5 \mathrm{k}-\mathrm{o}$ ). They were analyzed 


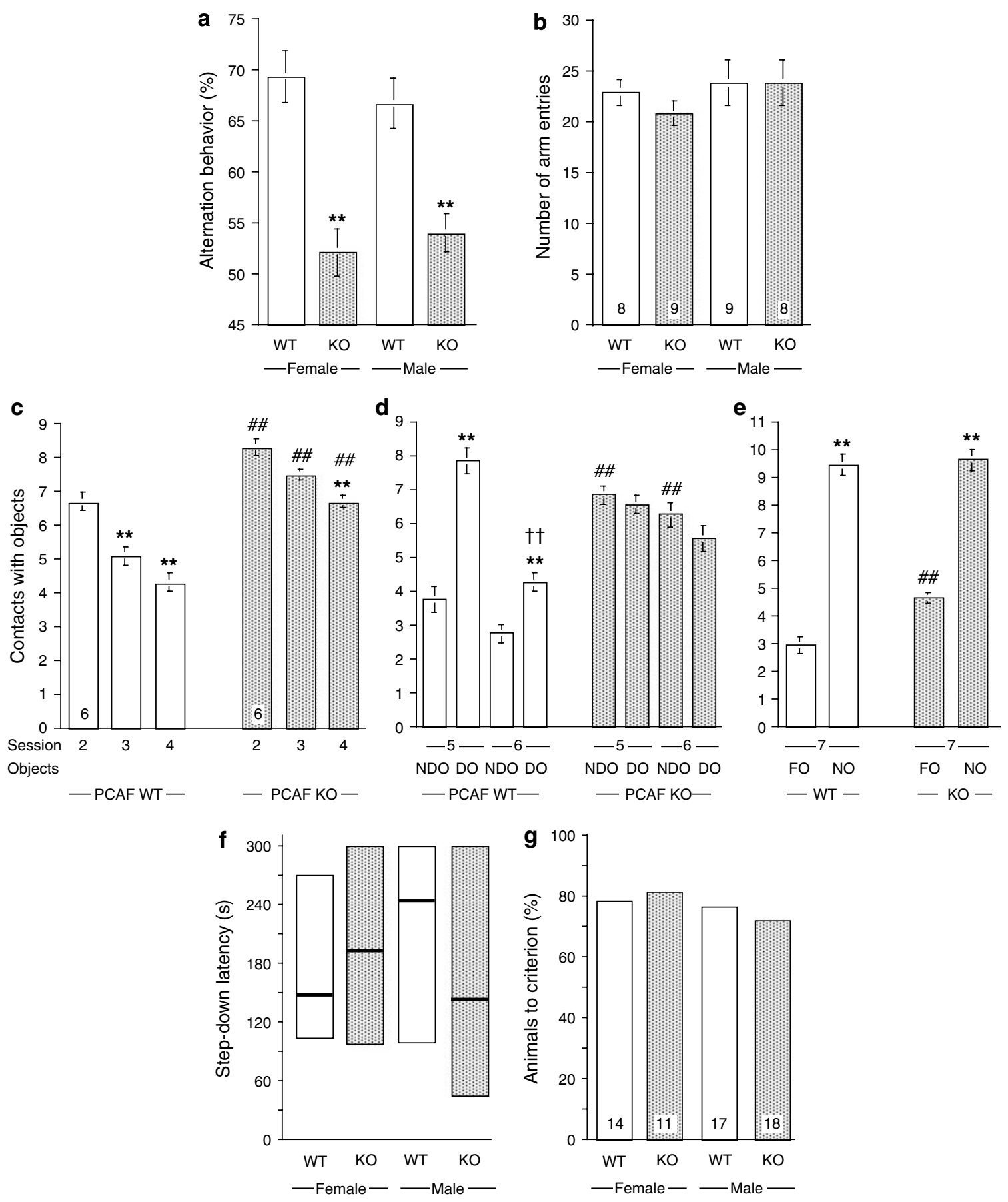

Figure 3 p300/CBP-associated factor (PCAF) knockout (KO) mice showed impairments of spontaneous alternation and object recognition, but not passive avoidance at 2 months of age. Responses of wild-type (WT) and PCAF KO mice in the spontaneous alternation test in the $Y$ maze: (a), alternation percentage; (b), total number of arms entered. ANOVA: (a) $F(1,30)=52.11, p<0.000$ I for substrain, $F<\mid$ for gender, $F=\mid$ for substrain $\times$ gender. (b) $F<1$ for substrain; $\mathrm{F}=$ I for gender; $\mathrm{F}<\mathrm{I}$ for the interaction. Responses in the object recognition test: (c), habituation to object exploration during sessions $2-4$; (d), reaction to change in object position during sessions 5 and 6; (e), reaction to a novel object during session 7. ANOVA: (c) $F_{(2,138)}=17.28, p<0.000$ I for session; $F_{(I, 138)}=61.23, p<0.0001$ for substrain, $F<I$ for the interaction; (d) $F_{(1,87)}=9.94, p<0.01$ for object, $F_{(1,87)}=27.80, p<0.0001$ for session, $F_{(1,87)}=21.53, p<0.0001$ for substrain, $F_{(1,87)}=4.16, p<0.05$ for the object $\times$ session interaction, $F_{(1,87)}=22.70, p<0.0001$ for the object $\times$ substrain interaction, $F_{(I, 87)}=56.86, p<0.05$ for the session $\times$ substrain interaction. (e) $F_{(I, 44)}=175.0, p<0.0001$ for object, $F_{(I, 44)}=4.45, p<0.05$ for substrain, $\mathrm{F}<\mathrm{I}$ for the object $\times$ substrain interaction. Responses in the passive avoidance test: $(\mathrm{f})$, step-down latency $(\mathrm{KW}=0.59, p>0.05)$; $(\mathrm{g})$, percentage of animals to criterion. The number of animals per group is indicated within columns in (b), (c), and (g). Abbreviations: NDO, nondisplaced objects; $\mathrm{DO}$, displaced objects; FO, familiar objects; NO, novel object. ${ }^{*}{ }^{*} p<0.01$ vs the respective control value. ${ }^{\# \#} p<0.01$ vs NDO value in the same session (Newman-Keuls test).

using two-way ANOVA with behavior and substrain as independent factors. In $\mathrm{CA} 1$, a significant effect was measured for behavior $\left(\mathrm{F}_{(1,8)}=12.48, p<0.01\right)$ and the interaction
$\left(\mathrm{F}_{(1,8)}=6.56, p<0.05\right.$; Figure 5e). In CA3, a significant effect was measured only for behavior $\left(\mathrm{F}_{(1,8)}=9.85, p<0.05\right.$; Figure $5 \mathrm{j}$ ). In the dendate gyrus, significant effects were 

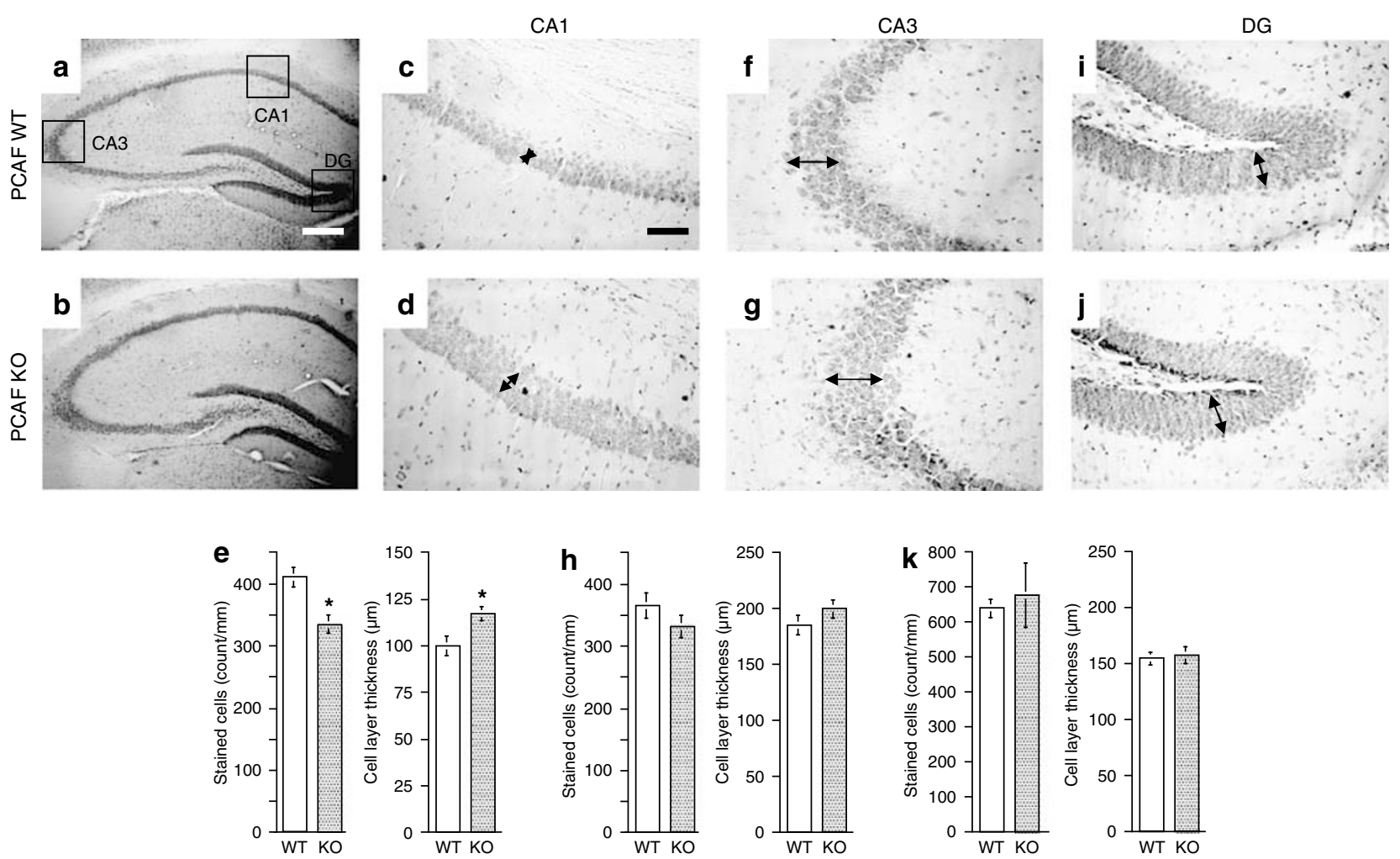

Figure 4 Morphological examination of the hippocampal formation of 2-month-old p300/CBP-associated factor (PCAF) knockout (KO) mice. Typical photomicrographs of the whole hippocampus ( $\mathrm{a}$ and b), CAI pyramidal neuronal layer ( $\mathrm{c}$ and d), CA3 layer ( $\mathrm{f}$ and $\mathrm{g}$ ) and the dentate gyrus ( $\mathrm{i}$ and $\mathrm{j}$ ) of wildtype (WT) (a, c, f, and i) and PCAF KO (b, d, g, and j) mice. (e, h, and k) Quantification of the layer thickness in the three hippocampal areas. Squares in (a) show the localization of CAI, CA3, and the dentate gyrus. Arrows in (c)-(j) point out where the thickness was measured using NIH software. Quantification was based on the mean value for 3-6 slices per animal and the number of animals indicated within columns in (e). Scale bar, $100 \mu \mathrm{m}$ in (a) and (b); $50 \mu \mathrm{m}$ in (c)-(j). ${ }^{*} p<0.05$ vs the WT value (Student's t-test).

observed for both behavior $\left(\mathrm{F}_{(1,8)}=80.0, p<0.0001\right)$ and substrain $\left(\mathrm{F}_{(1,8)}=7.04, p<0.05\right.$; Figure 50$)$, but not for the behavior $\times$ substrain interaction. In WT animals, the basal level of Fos-immunoreactive neurons was low and similar among areas. The behavioral examination led to a significant increase in Fos immunoreactivity in each area. In PCAF KO mice, the basal level of Fos-immunoreactive neurons was significantly higher than controls in CA1 and in the dentate gyrus and the behavior-induced increase was observed only in the CA3 and dentate gyrus areas (Figure 5). Alteration of hippocampal neuronal activation was thus observed in PCAF KO mice.

The level of ERK1/2 phosphorylation was measured in the hippocampus of WT and PCAF KO mice. Two batches of animals were assayed, ie mice submitted to the passive avoidance procedure and control animals, ie handled but behaviorally nontrained. Trained animals showed similar passive avoidance performances (step-down latency $151 \pm 61 \mathrm{~s}, n=5$, for WT mice $v s 190 \pm 45 \mathrm{~s}, n=4$, for PCAF KO mice). The shock-induced stress cannot be differentially analyzed from the learning paradigm since PCAF KO mice also showed an exaggerated response to stress, as reported thereafter. Therefore, we chose to analyze MAP kinase activation immediately after the retention session, at a time point where the memory retrieval process can be more selectively measured. Animals were therefore killed immediately after the retention session. As shown in Figure 6b, the behavioral testing induced a significant increase in ERK1 phosphorylation in WT, but not in PCAF KO mice. The behavioral procedure did not significantly affect the level of ERK2 phosphorylation in both WT and PCAF KO mice (Figure 6c).

\section{Emotional State and Response to Stress of PCAF KO Mice}

The response to acute stress of PCAF KO mice was first tested in the forced swimming stress test (Figure 7). During the initial session, PCAF KO showed an increased immobility duration over the 6-min trial. Indeed, after the second minute, the immobility duration shown by PCAF $\mathrm{KO}$ was significantly higher than that of WT control animals (Figure 7a). The analysis of the immobility duration, minute per minute, showed that PCAF KO mice remained immobile an average of $40 \mathrm{~s} / \mathrm{min}$ after the second minute, ie significantly more than WT, about $20 \mathrm{~s} /$ min (Figure $7 \mathrm{~b}$ ). During the second day session, mice developed a marked response to the learned stressful situation by showing increased immobility duration. However, PCAF KO still showed an increased response as compared to WT (Figure 7c). Indeed, the immobility duration shown by PCAF KO was not significantly different from that of WT (Figure 7c). The analysis of the immobility duration minute per minute showed that both PCAF KO and WT mice 

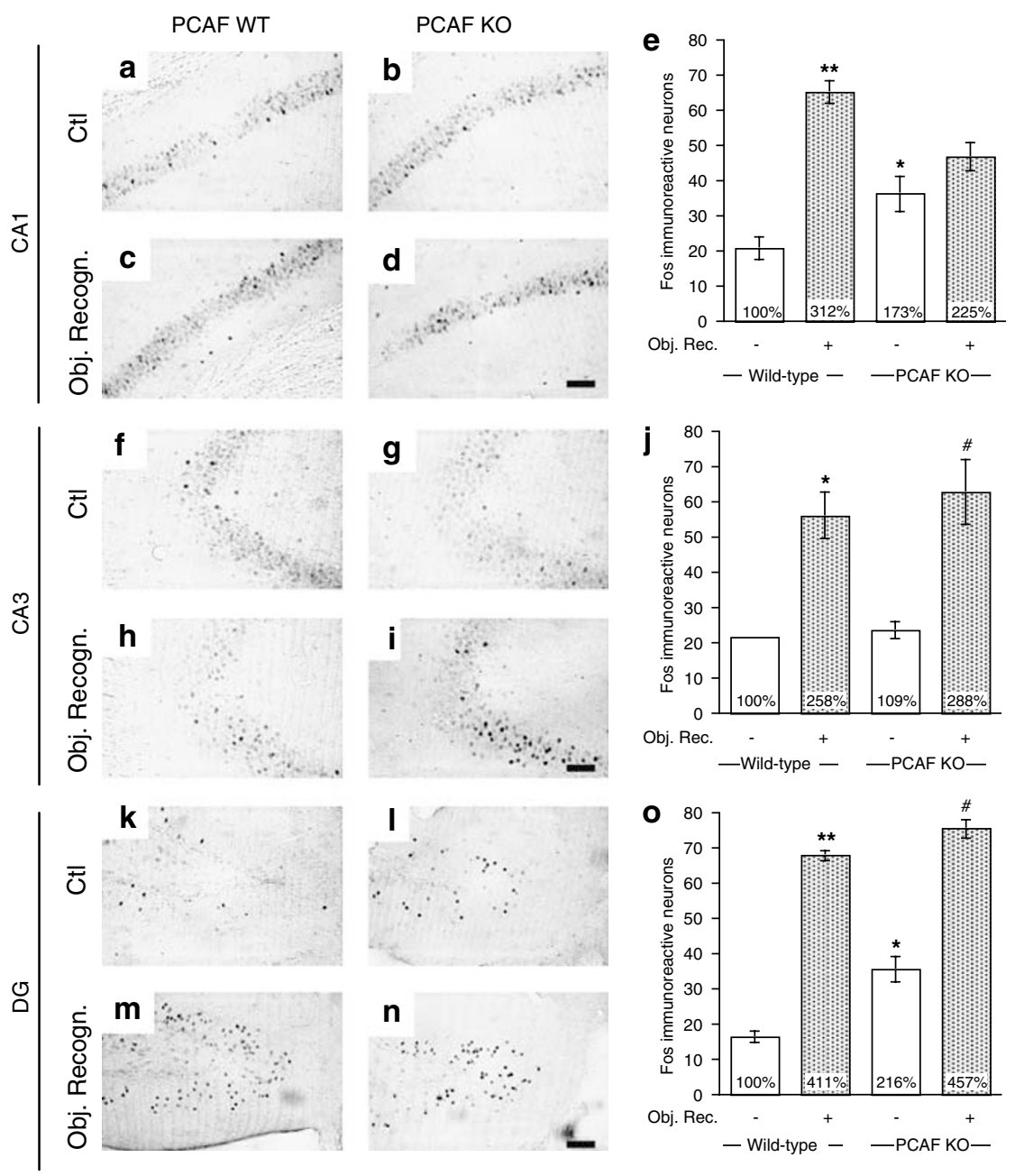

Figure 5 Two-month-old p300/CBP-associated factor (PCAF) knockout (KO) mice showed increased basal level of hippocampal Fos expression that altered training-induced neuronal activation, after an object recognition task. WT or PCAF KO male mice were used in control conditions (Ctl) or after behavioral examination in the object recognition procedure (Obj. Recogn.). The latter animals were killed I $\mathrm{h}$ after the task. The left panel shows typical photomicrographs of Fos immunoreactivity in the CAI (a-d), CA3 ( $f-i)$, and dendate gyrus areas $(k-n)$ of the hippocampus in WT and PCAF KO mouse brains. (e), (j), and (o), the right panel shows average Fos immunoreactivity density measurements. Data were expressed as percentage of levels in control WT mice, as shown within the columns. Scale bars, $100 \mu \mathrm{m}$. ANOVA: $F_{(I, 8)}=12.48, p<0.01$ for behavior, $F<I$ for substrain, $F_{(I, 8)}=6.56, p<0.05$ for the interaction. ${ }^{*} p<0.05,{ }^{*} * 0<0.01$ vs the CtI WT mouse value, ${ }^{\#} p<0.05$ vs the CtI PCAF KO mouse value (Newman-Keuls test).

tended to remain immobile an average of $40 \mathrm{~s} / \mathrm{min}$ and a significant difference between PCAF KO and WT animals was only measured during the fourth minute (Figure $7 \mathrm{~d}$ ). The cumulative immobility duration shown by the mice during the last $5 \mathrm{~min}$, which is classically used to screen for antidepressant-like activity, confirmed that PCAF KO mice remained, on day 1 , significantly more immobile than WT controls and showed that the level of immobility was comparable to the level reached by WT on day 2 (Figure 7e). Moreover, PCAF KO failed to show an augmentation in the global immobility score between days 1 and 2 .

PCAF KO animals were also tested in the conditioned fear stress test (Figure 8). This test includes anxiety impact in response to stress, since animals are retested in a stressorfree situation. As shown in Figure 8a, nonshocked PCAF KO mice exhibited a significantly lower ambulatory activity in the arena, as shown over the first $6 \mathrm{~min}$ of the session on day 1. Both WT and PCAF KO mice exhibited lower ambulatory activity when submitted to foot shocks
(Figure 8b), but PCAF KO mice still exhibited significantly lower activity. Indeed, significant differences were measured at all measured time points. No difference among groups was measured on the test day for nonshocked animals (Figure 8c). However, shocked PCAF KO showed very low ambulatory activity, which was significantly lower than the response of control WT mice (Figure 8d). Global activity scores are presented in Figure $7 \mathrm{e}$ and f. Nonshocked PCAF KO mice showed significantly lower activity on day 1 , similar to that observed in WT or themselves on day 2 (Figure 8e). This observation, which reflected an anxious reactivity to novelty, is in line with the increased object exploration measured during the first sessions in the object recognition test. In shocked animals, PCAF KO showed a lower activity than WT animals on day 1 during the shock application, but a significant stress-induced decrease in ambulatory activity was still observed between days 1 and 2 (Figure 8f). In particular, on day 2, PCAF KO mice showed significantly lower activity as compared to WT mice. 

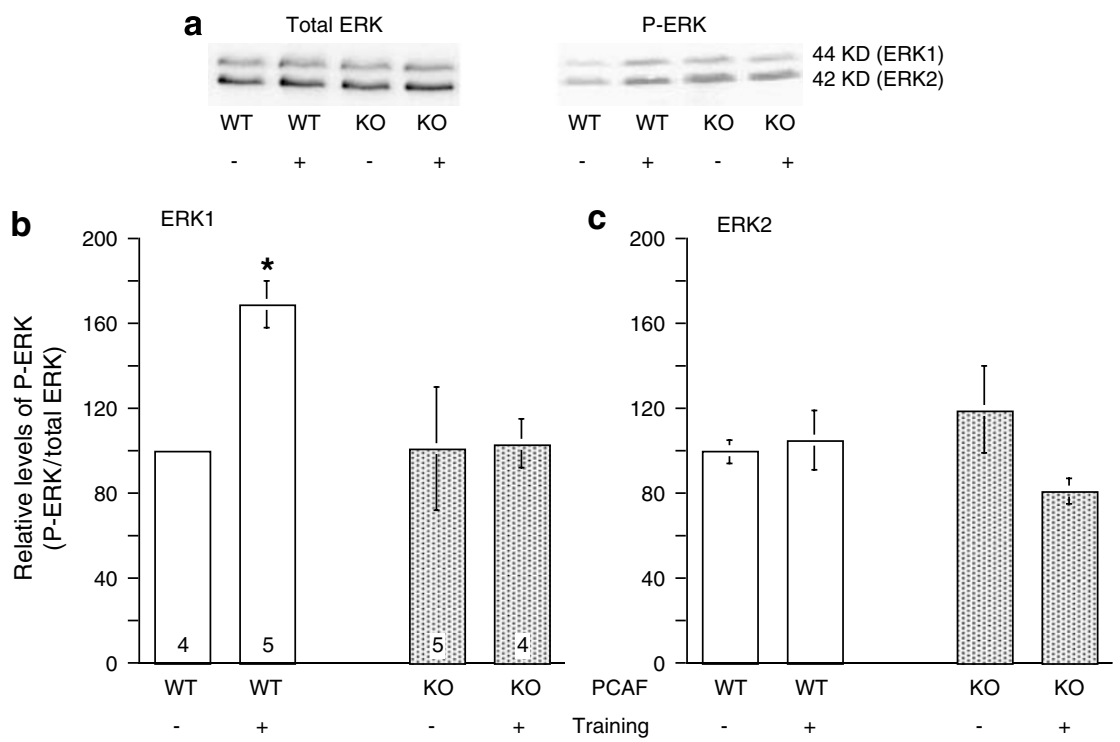

Figure 6 p300/CBP-associated factor (PCAF) knockout (KO) mice showed altered phosphorylation of ERKI after passive avoidance training. (a) Representative picture showing the effect of training in wild-type (WT) and PCAF KO mice on phosphorylated ERKI and ERK2 protein expression (-, control animals; +, trained animals). (b and c) Ratios of phosphorylated ERK (P-ERK) to total ERK in the hippocampus, expressed as a percentage of the control WT mice value. Animals were trained in the passive avoidance test and retention was performed after $24 \mathrm{~h}$. Trained animals and naive controls were immediately killed and hippocampi were used for western blot analyses. The number of animals per group is indicated within columns in (b). Data were analyzed using a Kruskal-Wallis ANOVA. *p $<0.05$ vs control WT mice value (Dunn's test).

These results showed that PCAF KO mice presented a marked hyperresponsiveness to acute stress. Hyperactivation of the hypothalamo-pituitary-adrenal axis results notably in an augmentation of corticosterone from the adrenals. Plasma corticosterone levels were therefore measured in control WT and PCAF KO mice and in animals submitted to $15 \mathrm{~min}$ forced swimming. Nonstressed PCAF KO mice presented a highly significant $+200 \%$ increase in corticosterone contents: $278 \pm 10 \mathrm{ng} / \mathrm{ml} \quad(n=6) \quad v s$ $89 \pm 13 \mathrm{ng} / \mathrm{ml}(n=6)$ for WT $(p<0.01)$. The 15 -min forced swimming stress significantly increased corticosterone levels in WT $(275 \pm 22 \mathrm{ng} / \mathrm{ml}, n=6, p<0.01 v s$ nonstressed animals) and also in KO mice $(345 \pm 17 \mathrm{ng} / \mathrm{ml}, n=6$, $p<0.01 v s$ nonstressed PCAF KO and $v s$ stressed WT animals). Therefore, PCAF KO mice remained able to respond to stress but presented, in nonstressed conditions, similar corticosterone levels as stressed WT animals.

Constitutively high corticosterone levels may lead to a chronic depressive state. Therefore, a putative anhedonic state, usually associated with depression, was investigated. The spontaneous drinking preference for a sucrose solution was measured over 9 days. As shown in Supplementary Figure 2a, the tap water and sucrose intake patterns did not differ among substrains. Moreover, both WT and PCAF KO animals gradually developed a high (about $80 \%$ ) sucrose preference after a couple of days (Supplementary Figure 2b). The PCAF KO mice are thus not spontaneously depressed.

\section{Age-Related Memory Impairment Patterns in PCAF KO Mice}

Male mice were tested again at 6 months of age and female mice at 12 months of age, to determine whether the memory impairment would evolve with age. We examined these middle-aged animals in the open-field, water-maze learning and passive avoidance tests.

At 6 months of age, PCAF KO animals showed significant increases in the latency to leave the center ring of the arena, and in the numbers of rearing and grooming reactions (Table 2). In addition, a nonsignificant trend toward increased total locomotion, locomotion at the center of the arena, and exploratory speed was noted (Table 2). At 12 months of age, PCAF KO mice still showed a significantly increased number of rearing reactions and a significant decrease in the locomotion at the center of the arena (Table 2). A nonsignificant trend toward increased total locomotion and exploratory speed was still observed (Table 2). It was thus concluded that PCAF KO mice developed some perturbations in exploration behavior and stereotyped behaviors with age, which may contribute to the expression of cognitive deficits.

In the water-maze test, both 6-month-old WT and PCAF KO mice showed decreased latencies in finding the platform during acquisition of the fixed platform location (Figure 9a). However, latencies shown by PCAF KO animals were augmented as compared with WT mice between trials 2 and 6 (Figure 9a). Moreover, during the probe test, only WT animals preferentially swam in the T quadrant and not PCAF KO (Figure 9b), indicating that PCAF KO mice developed long-term memory deficits. WT mice showed a classical profile for the acquisition of a daily changing platform location (Figure 9c). A substantial decrease between the first and second trial latency was measured. PCAF KO animals showed impaired short-term memory, ascribed to a significant increase in the trial 2 latency $(p<0.05$; Figure 9c). Note that the deficit in long-term memory observed in this spatial procedure in the watermaze test was not reproduced in a contextual procedure, ie a passive avoidance test (Figure 9d and e). Neither the 

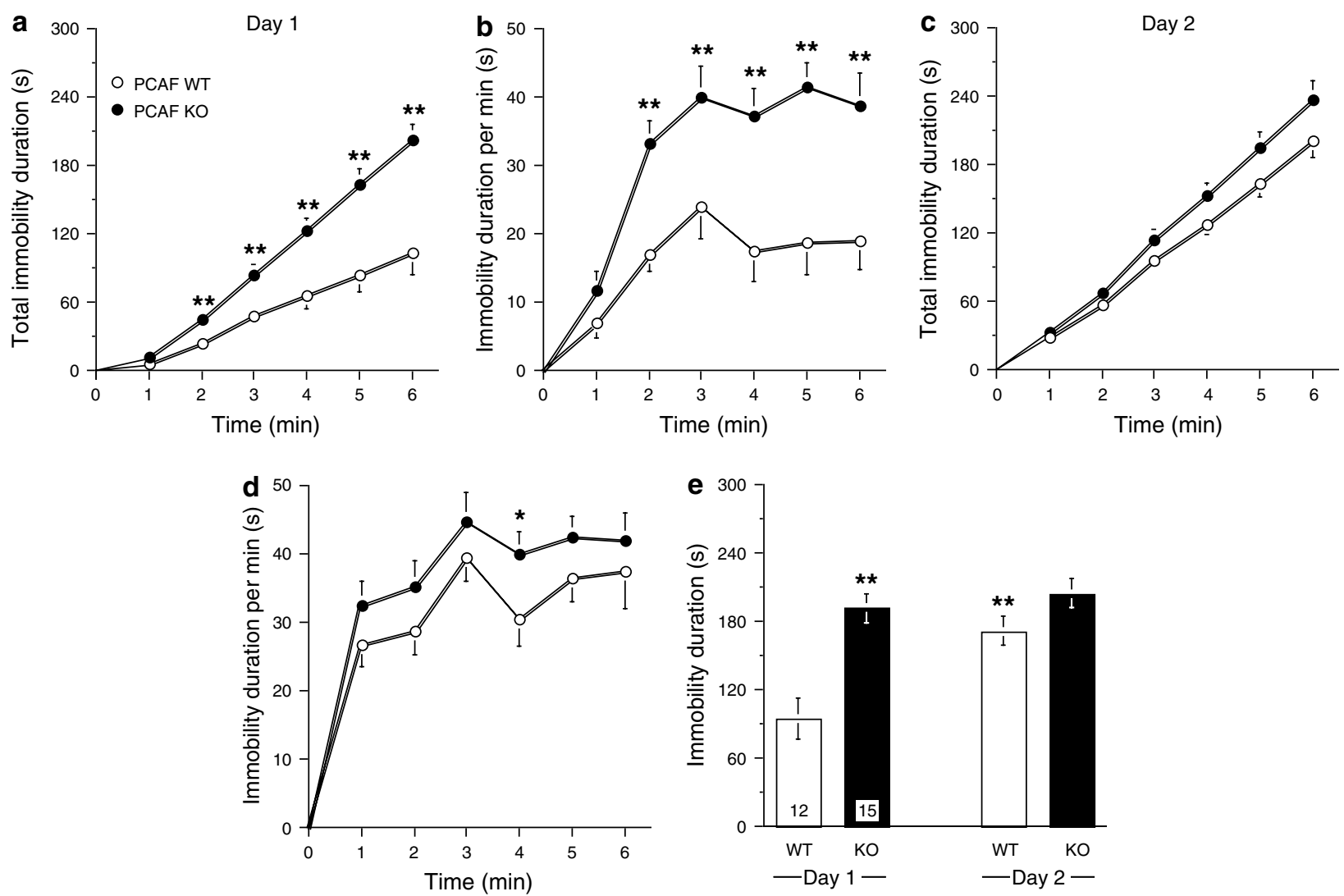

Figure 7 p300/CBP-associated factor (PCAF) knockout (KO) mice showed an increased behavioral despair response in the forced swimming test. Responses of 2-month-old wild-type (WT) and PCAF KO mice in the forced swimming test: ( $a$ and b) time course of the immobility duration and distribution of the immobility minute per minute, during the first 6 min of the session on day I; (c and d) time course of the immobility duration and distribution of the immobility minute per minute, during the 6 min of the session on day 2; (e) global immobility score between 1 and 6 min. The number of animals per group is indicated within columns in (e). In (a)-(d), data were analyzed using a two-way ANOVA with substrain and time as independent factors: (a) $F_{(5,150)}=51.1, p<0.0001$ for time, $F_{(1,150)}=64.8, p<0.0001$ for substrain, $F_{(5,150)}=5.58, p<0.0001$ for the time $\times$ substrain interaction; (b) $F_{(5,150)}=8.83, p<0.000$ I for time, $F_{(I, 150)}=54.1, p<0.000$ I for substrain, $F_{(5,150)}=1.28, p>0.05$ for the time $\times$ substrain interaction; $(c) F_{(5,150)}=89.8$, $p<0.000$ I for time, $F_{(1.150)}=13.3, p<0.001$ for substrain, $F<\mid$ for the time $\times$ substrain interaction; $(d) F_{(5.150)}=3.26, p<0.01$ for time, $F_{(1.150)}=8.10$, $p<0.0$ I for substrain, $\mathrm{F}<\mathrm{I}$ for the interaction. $* p<0.05$ vs WT value for the same time. In (e), data were analyzed using a two-way ANOVA with day and substrain as independent factors: $F_{(1,50)}=9.85, p<0.01$ for day, $F_{(1,50)}=19.97, p<0.000$ I for substrain, $F_{(1,50)}=4.78, p<0.05$ for the interaction. *** $p<0.0$ I vs WT value on day I (Newman-Keuls test).

step-down latency (Figure 9d) nor the percentage of animals to criterion (Figure 9e) showed a statistically significant difference between WT and PCAF KO animals.

The 12-month-old PCAF KO animals presented higher impairment levels. In the water-maze test, only WT, but not PCAF KO, mice showed lower latencies (Figure 9f). Moreover, latencies shown by PCAF KO animals appeared to be significantly higher than that observed for WT mice between trials 5, 6, and 8 (Figure 9f). During the probe test, only WT animals preferentially swam in the T quadrant, contrary to PCAF KO (Figure 9g). Moreover, the difference in time spent in the $\mathrm{T}$ quadrant between each group was significant. In the short-term memory procedure, WT mice acquired the daily changing platform location, since a marked decrease in latencies was measured (Figure 9h). PCAF KO animals showed impaired short-term memory, since no significant decrease in latency was observed over the swimming trials. The latencies measured for trials 2-5 were significantly higher as compared to WT animals (Figure 9h). Passive avoidance deficits were measured for 12-month-old PCAF KO animals, both in terms of step-down latency (Figure 9i) and percentage of animals to criterion (Figure 9j), indicating that contextual long-term memory was also affected at this age.

\section{DISCUSSION}

The PCAF histone acetylase shares a double function, acting as a transcriptional coactivator and as a HAT, and contributing to transcriptional activation during gene transcription by allowing nucleosome acetylation and chromatin structure remodeling (Ogryzko et al, 1996; Yamauchi et al, 2000; Korzus et al, 2004). The targeted inactivation of PCAF in CD1 mice failed to affect the level of histone $\mathrm{H} 3$ and $\mathrm{H} 4$ acetylation, suggesting that other(s) $\mathrm{HAT}(\mathrm{s})$-mediated $\mathrm{H} 3$ acetylation on Lys ${ }^{14}$ and $\mathrm{H} 4$ acetylation on Lys ${ }^{8}$ may be GCN5. However, it cannot be excluded that histone acetylation on specific genes may be impaired in PCAF KO mice, resulting in a complex phenotypic pattern. PCAF KO animals were viable, reached sexual maturity, and could be bred normally, contrary to GCN5 or 

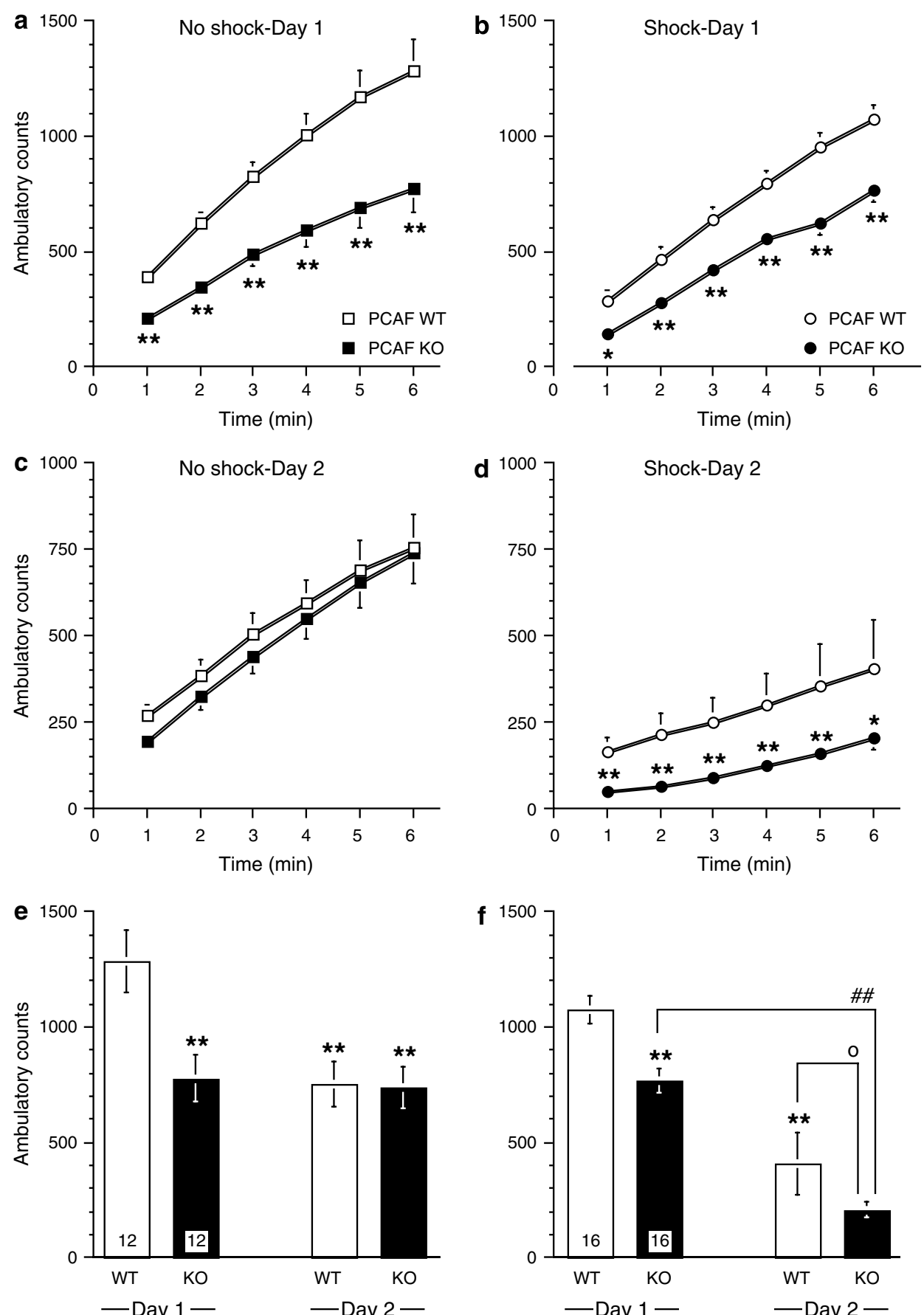

Figure 8 p300/CBP-associated factor (PCAF) knockout (KO) mice showed an increased freezing response in the conditioned fear stress test. Responses of 2-month-old wild-type (WT) and PCAF KO mice in the conditioned fear stress test: time course of ambulatory activity during the first 6 min of the session on day I for nonshocked control animals in (a) and shocked animals in (b). Time course of the ambulatory activity during 6 min of the session on day 2 for nonshocked control animals in (c) and shocked animals in (d). Global activity score over 6 min for nonshocked control animals in (e) and shocked animals in (f). The number of animals per group is indicated within columns in (e) and (f). In (a)-(d), data were analyzed using a two-way ANOVA with substrain and time as independent factors: (a) $F_{(5,132)}=24.9, p<0.000$ I for time, $F_{(1,132)}=66.0, p<0.000$ I for substrain, $F_{(5,132)}=1.24, p>0.05$ for the time $\times$ substrain interaction; (b) $F_{(5,180)}=63.2, p<0.000$ I for time, $F_{(1,180)}=77.0, p<0.000$ I for substrain, $F_{(5,180)}=1.21$, $p>0.05$ for the time $\times$ substrain interaction; (c) $F_{(5,132)}=17.3, p<0.0001$ for time, $F_{(1,132)}=1.54, p>0.05$ for substrain, $F_{(5,132)}=0.05, p>0.05$ for the time $\times$ substrain interaction; (d) $F_{(5,180)}=8.36$, $p<0.0001$ for time, $F_{(1,180)}=63.6, p<0.0001$ for substrain, $F_{(5,180)}=0.42, p>0.05$ for the time $\times$ substrain interaction. $* p<0.05$, *** $p<0.01$ vs $W T$ value for the same time. In (e) and ( $f$ ), data were analyzed using a two-way ANOVA with substrain and day as independent factors: (e) $F_{(I, 44)}=6.01, p<0.05$ for substrain, $F_{(I .44)}=7.15, p<0.05$ for day, $F_{(1,44)}=5.37, p<0.05$ for the substrain $\times$ day interaction; $(f) F_{(I .60)}=20.6, p<0.0001$ for substrain, $F_{(I .60)}=121.8$, $p<0.000$ I for day, $F_{(I, 60)}=0.86, p>0.05$ for the substrain $\times$ day interaction. ${ }^{*} * p<0.0$ I vs WT value on day I, ${ }^{\# \#} p<0.05$ vs day I value, ${ }^{\circ} p<0.05$ vs $W T$ value on the same day (Newman-Keuls test). 
Table 2 Behavioral Parameters of 6- and 12-Month-Old PCAF KO and WT Mice in the Open-Field Test

\begin{tabular}{|c|c|c|c|c|}
\hline \multirow[b]{2}{*}{ Parameter } & \multicolumn{2}{|c|}{ 6-month-old male mice } & \multicolumn{2}{|c|}{ I 2-month-old female mice } \\
\hline & WT & PCAF KO & WT & PCAF KO \\
\hline Latency to start (s) & $1 \pm 0$ & $9 \pm 2 *$ & $0 \pm 0$ & $1 \pm 0$ \\
\hline Locomotion (m) & $27.6 \pm 2.4$ & $35.8 \pm 1.9$ & $14.6 \pm 2.2$ & $20.3 \pm 3.3$ \\
\hline Immobility (s) & $98 \pm 25$ & $93 \pm 7$ & $285 \pm 36$ & $243 \pm 31$ \\
\hline Rearing & $14 \pm 2$ & $52 \pm 7$ *** & $8 \pm 3$ & $20 \pm 2 * *$ \\
\hline Grooming & $5 \pm 0$ & $24 \pm 2 * * *$ & $5 \pm 1$ & $5 \pm 1$ \\
\hline Defecations & $2 \pm 0$ & $3 \pm 0$ & $4 \pm 1$ & $6 \pm 0$ \\
\hline$n$ & 8 & 4 & 12 & 13 \\
\hline
\end{tabular}

Abbreviations: KO, knockout; PCAF, p300/CBP-associated factor; WT, wild type.

$* p<0.05, * * 0.01$ vs the age-matched WT group, Student's $t$-test.

CBP homozygous KO animals (Yamauchi et al, 2000). However, behavioral and physiological examination showed marked learning and memory impairments and an exaggerated response to acute stress. Moreover, contrary to heterozygous CBP KO models, PCAF KO mice initially showed impairments in short-term memory that affected long-term memory only later in life.

Behavioral phenotyping of 2-month-old PCAF KO mice using tests to assess general activity and memory functions initially showed that the general activity of PCAF KO mice resembled that of WT controls. Open-field examination, in particular, showed similar measures for parameters related to locomotion, exploration, emotional response, and stereotyped behaviors. Second, no sex-related difference was noted regardless of the behavioral parameter examined.

However, we observed marked alterations in the shortterm memory component, shown by deficits in spontaneous alternation, significant delay in escape latencies during water-maze acquisition sessions, and significant increases in swimming latencies during the procedure involving a daily changing platform position (Meunier and Maurice, 2004). The delay in acquisition of the platform position was related to short-term memory impairment. Indeed, repetitive training of a fixed platform position involved shortterm memory among trials within the same training day, and reference memory among training days. Global impairment of both short-term and reference spatial memory components will lead to learning impairments that were observed during the probe test. Impairment of the short-term or reference memory components may induce a significant delay in the acquisition profile due to worsening of the learning quality during each training trial that, however, could be attenuated through repetition of learning trials without any obvious probe test deficit. Recognition memory, another form of short-term memory, was markedly impaired in PCAF KO mice. When placed in the presence of four different objects, PCAF KO mice showed an increased level of interaction with objects that altered the quality of habituation and blocked the correct reaction to object position changes. Perturbations in reaction to novelty, exploration, or short-term memory may be involved in the observed pattern. Interestingly, during session 7, PCAF KO mice displayed a correct reaction to the presence of the novel object, ultimately suggesting that animals become familiar with the three other objects but also that mnesic processes selectively involving the hippocampal formation, such as change in object position, were more altered than less hippocampus-dependent processes, such as the discrimination between novel and familiar objects (Ennaceur and Delacour, 1988). Finally, short-term memory impairment could likely explain the deficits in object recognition observed in PCAF KO mice.

Figure 9 Memory impairments in p300/CBP-associated factor (PCAF) knockout (KO) mice increased with age: place learning in the water maze (a-c, f-h), and passive avoidance performance (d, e, i, and j) for 6-month-old male (a-e) and I2-month-old female (f-j) mice. (a and f) Acquisition latencies for a fixed platform position in the water-maze test. Animals were submitted for 7 or 9 days to three swims per day, with an intertrial time interval of I0 min. ANOVA: (a) $F_{(6,55)}=18.66, p<0.000$ I for wild-type $(W T), F_{(6,27)}=11.90, p<0.00$ I for PCAF KO mice; $(f) F_{(8,98)}=29.69, p<0.00$ I for $W T, F_{(8,98)}=9.18, p>0.05$ for PCAF KO mice. * $p<0.05$, ** $p<0.01$ vs the control value during the same training trial (Dunnett's test). (b and $g$ ) The platform was removed and animals were submitted to a $60 \mathrm{~s}$ probe test I $\mathrm{h}$ after the last swimming trial. The presence in each quadrant was measured. Quadrants: $T$, training; $A L$, adjacent left; $O$, opposite; AR, adjacent right. The number of animals per group is indicated in the T column. ANOVA: (b) $F_{(3.27)}=5.54, p<0.01$ for WT, F $<1$ for $P C A F K O$ mice; (g) $F_{(3,44)}=8.61, p<0.000$ I for $W T, F_{(3,44)}=1.85, p>0.05$ for PCAF KO mice. ${ }^{*} p<0.05$, $* * * 0.01$ vs the time spent in the T quadrant, ${ }^{\#} p<0.05$ vs PCAF WT value (Dunnett's test). (c and h) Short-term memory deficits were measured using the daily changing platform position procedure: swimming latency profiles among the five trials. ANOVA: (c) $F_{(4,34)}=52.75, p<0.000$ I for WT, $F_{(4,19)}=48.05, p<0.000$ I for PCAF KO; (h) $F_{(4,49)}=2.83, p<0.05$ for WT, $F<1$ for PCAF KO mice. ${ }^{*} p<0.05$, ${ }^{*}$ p $p<0.0$ I vs the control value (Dunn's test). Passive avoidance behavior: ( $\mathrm{d}$ and $\mathrm{i}$ ) step-down latency; (e and $\mathrm{j}$ ) percentage of animals to criterion. The number of animals is indicated in (e and j). ${ }^{*} * p<0.0$ I vs WT data; Mann-Whitney's test in (i), $\chi^{2}$-test in (j). 
Long-term memory appeared, however, to be preserved in young PCAF KO mice. Animals could acquire the fixed position of the platform in the water maze and showed
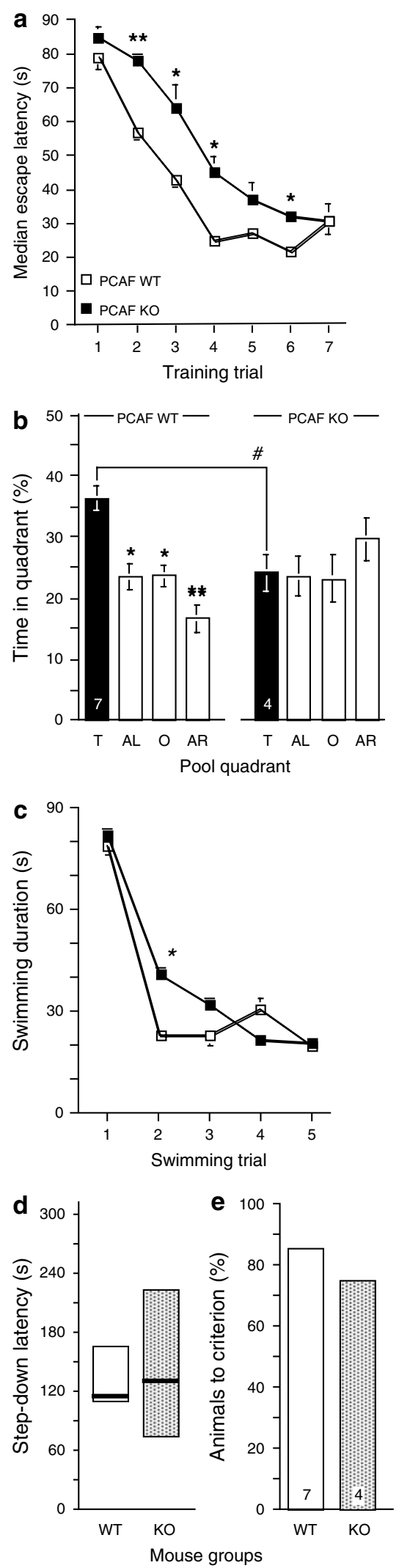

correct passive avoidance learning performances. The key finding of our study is thus that the memory impairment profile shown by PCAF KO mice differs from previously
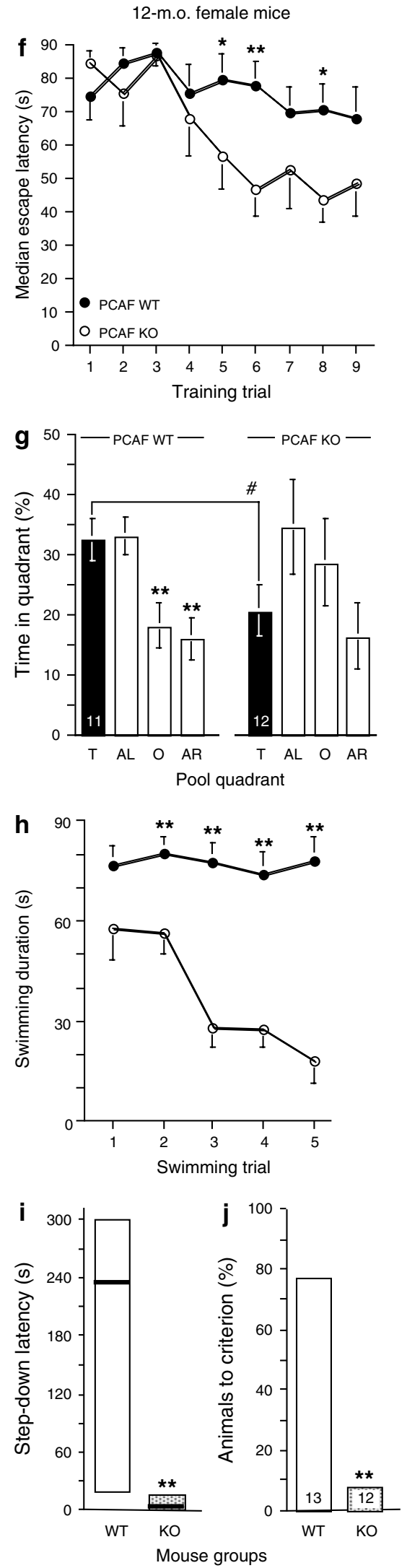
reported patterns in heterozygous $\mathrm{CBP} \mathrm{KO}$ and $\mathrm{CBP}\left(\mathrm{HAT}^{-}\right)$ animals. CBP KO mice showed long-term memory deficits, measured using passive avoidance, fear conditioning, and a long-term object recognition procedure (Oike et al, 1999; Alarcon et al, 2004). Short-term memory, measured using spontaneous alternation or a short-term memory version of the radial-arm maze task, was mainly preserved. However, Oike et al (1999) reported hypolocomotor activity in CBP KO mice, whereas Alarcon et al (2004) observed unaffected open-field parameters, anxiety responses, sensorimotor gating, and motor coordination responses. These differences could be attributed not only to the different inactivation strategies, but also to the different genetic backgrounds of the transgenic animals, C57BL/6 or C57BL/ $6 \times \mathrm{BALB} / \mathrm{C}$ hybrids (Oike et al, 1999; Alarcon et al, 2004). The selective alteration of long-term memory processes together with preservation of short-term memory in CBP KO mice suggested that memory consolidation, storage, and/or long-term retrieval, but not acquisition, are selectively affected, and that these animals may represent a model for the cognitive deficits in Rubinstein-Taybi syndrome (Oike et al, 1999; Alarcon et al, 2004). Mental retardation in Rubinstein-Taybi syndrome patients is characterized by low intelligence, a short attention span, and major difficulties in planning and executing motor skills (Gotts and Liemohn, 1977). Its modeling in animal tests could be assessed through general hypoactivity and learning impairment consistent with the deficits observed in CBP KO mice.

The differences observed in the behavioral phenotypes of CBP KO and PCAF KO animals suggest that the embryonic inactivation of PCAF, and the resulting alteration of gene expressions, directly affect the neuronal activation and rapid signaling involved in short-term memory. The hippocampus is critically involved in different forms of memory, including spatial and recognition memory. Morphological examination of the pyramidal cell layer morphology revealed significant cell loss associated with thickening of the CA1 area in PCAF KO mice. Changes in the CA3 area were much less marked and not significant. A more extensive morphofunctional study using specific markers is needed. However, this observation suggests that postnatal developmental alterations in PCAF KO mice have morphological consequences. We assessed two hippocampal-dependent physiological responses. First, Fos expression was measured in basal conditions and after activation by the object recognition procedure in WT and PCAF KO animals. Basal Fos expression increased in CA1 and the dendate gyrus in PCAF KO mice. Moreover, the increase in Fos expression induced by the behavioral procedure was significantly attenuated in the CA1 area, denoting a clear hyporesponsiveness of CA1 neurons. These observations were likely not related to morphological alterations in the cell layers, since the extent of the increase in basal Fos expression $(+74 \%)$ was higher than the differences in cell density $(-18 \%)$. Moreover, this pattern was also observed in the dentate gyrus. Second, we analyzed the induction of MAP kinase activity by measuring phosphorylated ERK levels. PCAF KO mice showed similar levels of phosphoERK in basal conditions, whereas training-associated induction of phospho-ERK expression was observed in WT mice but not in PCAF KO mice. Animals underwent passive avoidance training, ie a procedure known to induce rapid and sustained ERK activation (Han and Kim, 2003; Lee et al, 2004). However, ERK induction, and particularly ERK1 phosphorylation, may not be a critical prerequisite for a preserved passive avoidance response (Selcher et al, 2001). Therefore, these experimental conditions were ideal because they highlighted that the neuronal activation involved in learning was impaired in PCAF KO animals although they showed no major deficit in behavioral response. Both modified responses in PCAF KO mice suggest that the absence of histone acetylation results in a complex pattern of deficits resulting from modification of gene expression levels under basal or stimulated conditions.

PCAF KO mice were retested at the behavioral level at older ages. Since mice were housed in groups and presented a high level of endogenous corticosterone, we were not surprised to find, particularly in male animals, a high tendency to fight with each other and to show a rapid progressive degradation in their general features. Male animals were therefore selected to be tested at 6 months of age and female animals at 12 months of age. Adult, but not senescent or even aged, animals were therefore considered. Short-term memory impairments were still observed in water-maze acquisition profiles and in the daily changing platform position procedure. However, long-term memory deficits were noteworthy, since the probe test performances were very poor for PCAF KO mice at both ages. Moreover, passive avoidance performances, which were unaltered at 6-month-old, showed marked deficits at 12-month-old, indicating that contextual long-term memory was affected. Therefore, memory impairments observed in PCAF KO mice changed with age toward long-term memory deficits affecting all components of the mnesic process, although emotionally reinforced memory appeared to be preserved longer.

Using a model of chronic social defeat, Tsankova et al (2006) recently showed that stress downregulated $B d n f$ transcripts III and IV and longlastingly increased histone H3 methylation at their respective promoters, therefore repressing gene transcription. Moreover, an antidepressant treatment with imipramine reversed this downregulation and resulted in increased acetylation of $\mathrm{H} 3$ histone at the promoters (Tsankova et al, 2006). These important results suggested a direct role of the chromatin state, with transcription being repressed through histone hypermethylation or activated through histone hyperacetylation, in the molecular effectors sustaining physiological allostasis to acute stress. Here we report that PCAF KO mice presented an increased response to acute stress. This was observed when mice were submitted to the forced swimming procedure, ie the most commonly used method for evaluating depression-like behavior and screening for antidepressant-like activity, and to the conditioned fear stress test, which is a psychological stress procedure that is sensitive to anxiolytics. This increased sensitivity was explained by augmented basal levels of plasma corticosterone in PCAF KO mice. Moreover, since PCAF KO were still able to respond to the stress, not only in terms of behavioral responses, but also in terms of corticosterone secretion, it appears that these mice presented a modification in their allostatic state, rather than a constitutive stressed phenotype. In agreement with this conclusion, PCAF KO 
mice did not show marked anhedonia, suggesting that they may not be constitutively depressive. PCAF thus seems to play a role in the chromatin remodeling involved in the regulation of gene expression controlling the emotional state and physiological allostasis. Moreover, PCAF could be involved in the histone acetylation sustaining acute antistress effects of antidepressant drugs. These observations pave the way for a new avenue of research on antistress or antidepressant compounds.

Noteworthy, corticosterone has been reported to affect memory processes. Repeated administration of corticosterone facilitates the acquisition of emotional memory, particularly by improving the consolidation and retention phases, ensuring the transition from short- to long-term memory (Sandi and Rose, 1994, 1997; Coburn-Litvak et al, 2003; Thompson et al, 2004). Therefore, the high corticosterone levels measured in PCAF KO mice may interfere with long-term memory, particularly as measured in the passive avoidance procedure. Consolidation processes facilitated by the high corticosterone tonus may compensate impaired learning, and account for the preserved passive avoidance performances as compared with alternation performance deficits. This may be particularly true for 6-month-old animals, since emotional learning appeared more preserved than spatial learning capacities.

The PCAF KO mouse thus appears to be a complex animal model exhibiting developmental and functional deficits that change during life. This study provides evidence that PCAF is involved in learning and memory processes and response to stress, but differently than CBP and related HATs. In particular, the PCAF KO mouse may constitute a unique model for screening antiamnesic and antidepressant activity, while taking the role of chromatin remodeling into account as a key mechanism controlling long-term adaptative changes associated with psychiatric conditions.

\section{ACKNOWLEDGEMENTS}

The behavioral analysis was a project (No. 01) of the CompAn animal behavior phenotyping facility (Montpellier, France). We thank Dr Monica Prieto (Montpellier) for technical advice in immunohistochemistry and Dr Karim Chebli and the animal facility of the Institut de Génétique Moléculaire (IGMM, IFR122, Montpellier) for mouse breeding.

\section{DISCLOSURE/CONFLICT OF INTEREST}

The authors declare that, except for income received from their primary employer, no financial support or compensation has been received from any individual or corporate entity over the past 3 years for research or professional service and there are no personal financial holdings that could be perceived as constituting a potential conflict of interest.

\section{REFERENCES}

Alarcon JM, Malleret G, Touzani K, Vronskaya S, Ishii S, Kandel ER et al (2004). Chromatin acetylation, memory, and LTP are impaired in $\mathrm{CBP}^{+/-}$mice: a model for the cognitive deficit in Rubinstein-Taybi syndrome and its amelioration. Neuron 42: 947-959.

Blanco JC, Minucci S, Lu J, Yang XJ, Walker KK, Chen $\mathrm{H}$ et al (1988). The histone acetylase PCAF is a nuclear receptor coactivator. Genes Dev 12: 1638-1651.

Chan HM, La Thangue NB (2001). p300/CBP proteins: HATs for transcriptional bridges and scaffolds. J Cell Sci 114: 2363-2373.

Coburn-Litvak PS, Pothakos K, Tata DA, McCloskey DP, Anderson BJ (2003). Chronic administration of corticosterone impairs spatial reference memory before spatial working memory in rats. Neurobiol Learn Mem 80: 11-23.

Ennaceur A, Delacour J (1988). A new one-trial test for neurobiological studies of memory in rats. 1: behavioral data. Behav Brain Res 31: 47-59.

Givalois L, Naert G, Rage F, Ixart G, Arancibia S, Tapia-Arancibia L (2004). A single brain-derived neurotrophic factor injection modifies hypothalamo-pituitary-adrenocortical axis activity in adult male rats. Mol Cell Neurosci 27: 280-295.

Gotts EE, Liemohn WP (1977). Behavioral characteristics of three children with the broad thumb-hallux (Rubinstein-Taybi) syndrome. Biol Psychiatry 12: 413-423.

Han Y, Kim SJ (2003). Memory enhancing actions of Asiasari radix extracts via activation of insulin receptor and extracellular signal regulated kinase (ERK) I/II in rat hippocampus. Brain Res 974: 193-201.

Hoshino M, Tagawa K, Okuda T, Murata M, Oyanagi K, Arai N et al (2003). Histone deacetylase activity is retained in primary neurons expressing mutant huntingtin protein. J Neurochem 87: 257-267.

Imhof A, Yang XJ, Ogryzko VV, Nakatani Y, Wolffe AP, Ge H (1997). Acetylation of general transcription factors by histone acetyltransferases. Curr Biol 7: 689-692.

Janknecht R (2002). The versatile functions of the transcriptional coactivators p300 and CBP and their roles in disease. Histol Histopathol 17: 657-668.

Jenuwein T, Allis CD (2001). Translating the histone code. Science 293: $1074-1080$.

Korzus E, Rosenfeld MG, Mayford M (2004). CBP histone acetyltransferase activity is a critical component of memory consolidation. Neuron 42: 961-972.

Kumar A, Choi KH, Renthal W, Tsankova NM, Theobald DE, Truong HT et al (2005). Chromatin remodeling is a key mechanism underlying cocaine-induced plasticity in striatum. Neuron 48: 303-314.

Lachner M, O’Sullivan RJ, Jenuwein T (2001). An epigenetic road map for histone lysine methylation. J Cell Sci 116: 2117-2124.

Lee JK, Choi SS, Lee HK, Han KJ, Han EJ, Suh HW (2004). Effects of MK-801 and CNQX on various neurotoxic responses induced by kainic acid in mice. Mol Cell 14: 339-347.

Levenson JM, O’Riordan KJ, Brown KD, Trinh MA, Molfese DL, Sweatt JD (2004). Regulation of histone acetylation during memory formation in the hippocampus. J Biol Chem 279: 40545-40559.

Levenson JM, Sweatt JD (2005). Epigenetic mechanisms in memory formation. Nat Rev Neurosci 6: 108-118.

Liu L, Scolnick DM, Trievel RC, Zhang HB, Marmorstein R, Halazonetis TD et al (1999). p53 sites acetylated in vitro by PCAF and p300 are acetylated in vivo in response to DNA damage. $\mathrm{Mol}$ Cell Biol 19: 1202-1209.

Lunyak VV, Burgess R, Prefontaine GG, Nelson C, Sze SH, Chenoweth J et al (2002). Corepressor-dependent silencing of chromosomal regions encoding neuronal genes. Science 298: $1747-1752$.

Martinez-Balbas MA, Bauer UM, Nielsen SJ, Brehm A, Kouzarides $\mathrm{T}$ (2000). Regulation of E2F1 activity by acetylation. EMBO J 9: 662-671. 
Masumi A, Wang IM, Lefebvre B, Yang XJ, Nakatani Y, Ozato K (1999). The histone acetylase PCAF is a phorbol-ester-inducible coactivator of the IRF family that confers enhanced interferon responsiveness. Mol Cell Biol 19: 1810-1820.

Meunier J, Maurice T (2004). Beneficial effects of the sigma ${ }_{1}\left(\sigma_{1}\right)$ receptor agonists igmesine and dehydroepiandrosterone against the learning impairments in rats prenatally exposed to cocaine. Neurotoxicol Teratol 26: 783-797.

Munshi N, Merika M, Yie J, Senger K, Chen G, Thanos D (1998). Acetylation of HMG I(Y) by CBP turns off IFN b expression by disrupting the enhanceosome. Mol Cell 2: 457-467.

Ogryzko VV, Kotani T, Zhang X, Schiltz RL, Howard T, Yang XJ et al (1998). Histone-like TAFs within the PCAF histone acetylase complex. Cell 94: 35-44.

Ogryzko VV, Schiltz RL, Russanova V, Howard BH, Nakatani Y (1996). The transcriptional coactivators p300 and CBP are histone acetyltransferases. Cell 87: 953-959.

Oike Y, Hata A, Mamiya T, Kaname T, Noda Y, Suzuki M et al (1999). Truncated CBP protein leads to classical RubinsteinTaybi syndrome phenotypes in mice: implications for a dominant-negative mechanism. Hum Mol Genet 8: 387-396.

Sandi C, Rose SP (1994). Corticosterone enhances long-term retention in one-day-old chicks trained in a weak passive avoidance learning paradigm. Brain Res 647: 106-112.

Sandi C, Rose SP (1997). Protein synthesis- and fucosylationdependent mechanisms in corticosterone facilitation of longterm memory in the chick. Behav Neurosci 111: 1098-1104.

Sartorelli V, Puri PL, Hamamori Y, Ogryzko VV, Chung G, Nakatani Y et al (1999). Acetylation of MyoD directed by PCAF is necessary for the execution of the muscle program. Mol Cell 4: 725-734.

Selcher JC, Nekrasova T, Paylor R, Landreth GE, Sweatt JD (2001). Mice lacking the ERK1 isoform of MAP kinase are unimpaired in emotional learning. Learn Mem 8: 11-19.

Steffan JS, Bodai L, Pallos J, Poelman M, McCampbell A, Apostol BL et al (2001). Histone deacetylase inhibitors arrest polyglutamine-dependent neurodegeneration in Drosophila. Nature 413: 739-743.

Thompson BL, Erickson K, Schulkin J, Rosen JB (2004). Corticosterone facilitates retention of contextually conditioned fear and increases CRH mRNA expression in the amygdala. Behav Brain Res 149: 209-215.

Tsankova NM, Berton O, Renthal W, Kumar A, Neve RL, Nestler EJ (2006). Sustained hippocampal chromatin regulation in a mouse model of depression and antidepressant action. Nat Neurosci 9: 519-525.

Tsankova NM, Kumar A, Nestler EJ (2004). Histone modifications at gene promoter regions in rat hippocampus after acute and chronic electroconvulsive seizures. J Neurosci 24: 5603-5610.

Varga-Weisz P (2005). Chromatin remodeling factors and DNA replication. Prog Mol Subcell Biol 38: 1-30.

Xu L, Lavinsky RM, Dasen JS, Flynn SE, McInerney EM, Mullen $\mathrm{TM}$ et al (1998). Signal-specific co-activator domain requirements for Pit-1 activation. Nature 395: 301-306.

Yamauchi T, Yamauchi J, Kuwata T, Tamura T, Yamashita T, Bae $\mathrm{N}$ et al (2000). Distinct but overlapping roles of histone acetylase PCAF and of the closely related PCAF-B/GCN5 in mouse embryogenesis. Proc Natl Acad Sci USA 97: 11303-11306.

Supplementary Information accompanies the paper on the Neuropsychopharmacology website (http://www.nature.com/npp) 